\title{
A two-level procedure for the global optimisation of the damping behaviour of composite laminated plates with elastomer patches
}

\author{
Marco Montemurro ${ }^{1}$, Angela Vincenti ${ }^{2}$, Yao Koutsawa ${ }^{3}$ and Paolo Vannucci ${ }^{4}$
}

\begin{abstract}
This work concerns a two-level procedure for the global optimum design of hybrid elastomer/composite modular structures. The goal of the procedure is the maximisation of the first $N_{f}$ modal loss factors of the structure, satisfying mechanical constraints on the weight and on the bending stiffness, feasibility constraints on the admissible moduli for the constitutive laminates, along with geometric constraints on the positions of the visco-elastic patches. At the first level of the procedure, the optimisation of the damping behaviour of the structure is carried out: the optimisation variables at this stage are the number of elastomer patches (modules), as well as their geometrical parameters (position, thickness and diameter), along with the material and geometric parameters of the composite laminated structure (elastic moduli, thickness of the laminate). The composite structure supporting the elastomer patches is thus optimised using a free-material approach, via the polar representation of $2 \mathrm{D}$ elasticity, and the second level of the optimisation consists in finding the laminate stacking sequence satisfying the optimal elastic moduli and thickness issued from the first step. The method is able to automatically determine the optimal number of modules and it does not need the introduction of any simplifying assumption. The proposed approach relies on one hand, on the application of the well-known Iterative Modal Strain Energy (IMSE) method for the evaluation of the dynamic response of the structure, and on the other hand on the use of the polar formalism for the representation of the elastic anisotropic behaviour of composite laminates as well as of a genetic algorithm as optimisation tool to perform the solution search. We will illustrate the application of our approach to the optimisation of the damping behaviour of a rectangular composite plate with a discontinuous aperiodic distribution of viscoealstic material. The numerical results show the effectiveness of the proposed strategy.
\end{abstract}

\section{Keywords}

Viscoelasticity, composite materials, modular structures, passive damping, optimisation, genetic algorithms

\footnotetext{
${ }^{1}$ Laboratoire d'Énergétique et de Mécanique Théorique et Appliquée, Mines Nancy, GIP-InSIC, Université de Lorraine, St-Dié-des-Vosges, France

${ }^{2}$ Institut d'Alembert, Université Pierre et Marie Curie Paris 6, Paris, France

${ }^{3}$ Centre de Recherche Public Henri Tudor, Luxembourg-Kirchberg, Luxembourg

${ }^{4}$ Laboratoire de Mathématiques de Versailles, Université de Versailles et St Quentin, Versailles, France

Corresponding author:

Marco Montemurro, Laboratoire d'Énergétique et de Mécanique Théorique et Appliquée, Mines Nancy, GIP-InSIC, Université de Lorraine, 27 rue d'Hellieule, 88100 St-Dié-des-Vosges, France.

Email: marco.monte1984@gmail.com
} 


\section{Introduction}

One of the most important challenges for automotive and aerospace industries is the reduction of noise pollution. In particular, the vibrations of the structural parts composing a system represent one of the major sources of noise: when structural components move, they produce noise. Moreover, due to their lightness and their high stiffness, composite materials are very sensitive to this phenomenon.

To this purpose, several passive solutions were proposed in the literature: among them a possible strategy consists in bonding elastomer patches on some well-chosen regions of the structure. Even though these solutions are proved to be quite effective (Zheng et al., 2004), they are often the result of a design process made a posteriori, that is to say the structure was already designed or even optimised with respect to some criteria, and a posteriori a solution for the reduction of noise is sought by the introduction of a fixed number of viscoelastic patches of variable size and position. If, on one hand, the bonding of new elastomer elements on the original structure improves its damping behaviour, on the other hand it increases its weight and it might reduce some mechanical properties.

To overcome such an issue, Kerwin was one of the first researchers which considered the design of sandwich beams including a priori viscoelastic layers (Kerwin, 1959). Solutions of this kind have been widely studied in the literature and have proven to be rather effective (Rao 1978; Lu et al. 1979; Chalak et al. 2011; Ghoneim and Karbouk 2001). Rather complete, though not exhaustive reviews on this subject can be found in (Nakra 1998; Chandra et al. 1999; Berthelot et al. 2008; Vasques et al. 2010a; Vasques et al. 2010b; Benjeddou 2001; Trinidade and Benjeddou 2002). Moreover, concerning both active and passive strategies for designing the damping properties of hybrid composite/elastomer sandwich plates the reader is addressed to (Araújo et al. 2009; Araújo et al. 2010; Araújo et al. 2012; Sher and Moreira 2013).

The majority of the studies conducted on hybrid elastomer/composite systems consider only the presence of continuous viscoelastic layers within the stack of the laminates composing the structure. To our best knowledge, the first researchers that investigate the effects of using discontinuous viscoelastic layers were Kristensen et al. (2008) who studied the problem of composite beams damped by discontinuous rubber layers (or patches) in two different cases: the case wherein the gaps between the patches are filled by composite material and the case wherein such gaps are unfilled. The authors highlight the importance of using continuous viscoelastic layers in order to obtain high damping capabilities. They considered a beam made of three layers (the bottom one represents the composite beam, the middle one the viscoelastic layer and the top one the so-called constrained stiffer layer) and they showed that "cutting" away parts of the rubber layer in particular regions of the structure, e.g. in the regions where the loads and/or the supports have to be applied, does not allow to ameliorate the damping capabilities of the system, but, on the contrary it might induce a broad reduction of such capabilities, depending on the region where the material is cut away. In addition, they asserted that an important parameter involved into the mechanism of dissipation of the vibratory energy is the thickness of the stiffer constrained layer rather than the thickness of the rubber one. They showed that, when keeping constant the thickness of the rubber ply and increasing the thickness of the stiffer layer, shear strains are forced into the viscoelastic ply which are responsible for the energy dissipation. Finally, they showed that the case of the beam with discontinuous rubber layers having gaps filled by composite material show better damping capabilities than those of the case with unfilled gaps.

Despite the work of Kristensen et al. (2008) presents several innovative points along with some interesting results, it is worth noting that the mathematical model adopted to describe the beam kinematic (based on the Timoshenko assumptions) does not take into account correctly the orthotropy of the elastic plies (in fact in that work the material behaviour of such plies is described only through two parameters, i.e. a Young's modulus $E$ and a shear modulus $G$ ). Moreover, the behaviour of the viscoelastic material is described using the well-known Kelvin-Voigt model which is not able to represent the relaxation behaviour of the rubber ply. In addition, the mechanical model employed in that work consider as primary source of damping only the mechanism associated to the shear deformations through-the-thickness of the viscoelastic material, thus neglecting the contribution of the other strain components. Together with the previous aspects, one can notice that the level of shear strains forced by the stiffer layer into the elastomer one is too high and hence the non-linearity of the model (either geometrical or material) must be taken into account in the calculations. Finally such a study is conducted considering only the influence of all design parameters on the first modal loss factor of the structure, ignoring the effect on the others loss factors associated to higher frequencies: it seems natural that the results found by the authors can be valid only considering the damping mechanism at low frequencies.

Recently, Le Maoût et al. (2011) studied the problem of laminated plates damped by using rubber patches: more precisely the main goal of that work is the maximisation of the modal loss factors of the structure (in a given range of frequencies) employing a periodical pattern of viscoelastic material. They considered a 
three-layer plate with the following design variables: thickness and orientation angle for the elastic plies and thickness, diameter and distribution for the rubber patches (they assumed that the patches are identical and equally spaced). The modal loss factors are evaluated according to the well-known Iterative Modal Strain Energy (IMSE) method. Also in this work two different cases were considered: the case wherein the gaps between the patches are filled by composite material and the case wherein such gaps are unfilled. In both cases the results (in terms of damping capability of the structure) are compared with those obtained using continuous viscoelastic plies. The authors showed that a simultaneous design of both elastic and viscoelastic properties of the structure can lead to obtain better damping capabilities with respect to the case of employing continuous viscoelastic layers.

Even though the work of Le Maoût et al. is characterised by some original aspects, the optimisation problem is solved introducing the aforementioned simplifying assumptions (patches identical and equally spaced, threelayer plate) which could affect the quality of the final solution.

The study presented in this work could be placed within the context of the works originally proposed by Le Maoût et al. and can be seen as a generalisation and also as an extension of those works. In particular we propose a global optimisation technique for the design of damping properties of hybrid elastomer/composite modular structures, i.e. composites laminated plates equipped with bonded elastomer patches. The goal of the procedure is to maximise the first $N_{f}$ modal loss factors of the structure subject to various constraints: on the flexural stiffness, on the weight of the plate, on the material design variables describing the behaviour of the composite plate and, finally, on the position of the patches over the composite plate.

The problem considered here belongs to the class of design problems of modular systems. The hybrid elastomer/composite structure studied here has two different types of modules. The modules of the first type are the patches. All the patches are modules because they have the same function and geometry, but not necessarily the same size (diameter and thickness) nor the same position. In addition also the number of patches is included among the design variables of the optimisation problem. The modules of the second type are the layers: all the layers, composing the composite plate, are identical (same constitutive material and same thickness), but normally they are differently oriented.

We adopt a two-level procedure for the global optimum design of the hybrid structure. This procedure has already been employed to treat other optimisation problems concerning modular composite structures, see (Montemurro et al. 2012a; Montemurro et al. 2012b) for more details. At the first level of the procedure, the optimal design of the hybrid elastomer/composite structure in terms of its damping capabilities is carried out, the design variables in this phase being the geometrical parameters characterising each patch-module (number, position, thickness and diameter) along with the material and geometric parameters describing the composite plate that is considered as an equivalent single layer (namely such parameters are the laminate polar moduli and the total thickness of the plate). At the second level of the procedure we look for at least one stacking sequence corresponding to the optimal elastic moduli and thickness found for the composite plate at the first level. The proposed approach relies on one hand, upon the dynamic response of the structure in terms of natural undamped frequencies and modal loss factors which are evaluated using the IMSE method, and on the other hand on the use of the polar formalism for the representation of the elastic anisotropic behaviour of composite laminates as well as of the genetic code BIANCA with crossover and mutation between different species (Montemurro et al. 2012b; Montemurro et al. 2013; Vincenti et al. 2010) as optimisation tool to perform the solution search.

The paper is organised as follows: the mechanical problem considered in the study as well as the two-level strategy are introduced in Section 2.. The mathematical formulation of the first-level problem is detailed in Section 3. and the problem of determining a suitable laminate is formulated in Section 4.. A concise description of the FE model of the hybrid structure is given in Section 5., while in Section 6. we show the numerical results of the whole optimisation procedure. Finally, Section 7. ends the paper with some concluding remarks and perspectives.

\section{Optimal Design of composite plates with bonded elastomer patches}

\subsection{Description of the problem}

The optimisation procedure presented in this work is applied to the hybrid structure depicted in Figure 1.

Such a hybrid structure is composed of a laminated plate with surface-bonded viscoelastic patches. The length of the plate sides are $L=200 \mathrm{~mm}$ and $w=20 \mathrm{~mm}$, respectively. The plate is made of glass-epoxy laminae, whose material properties are taken from (Le Maoût et al., 2011) and listed in Table 1. Concerning the definition of the polar parameters of the constitutive layer that appear in Table 1 the reader is addressed to Section 3.. 


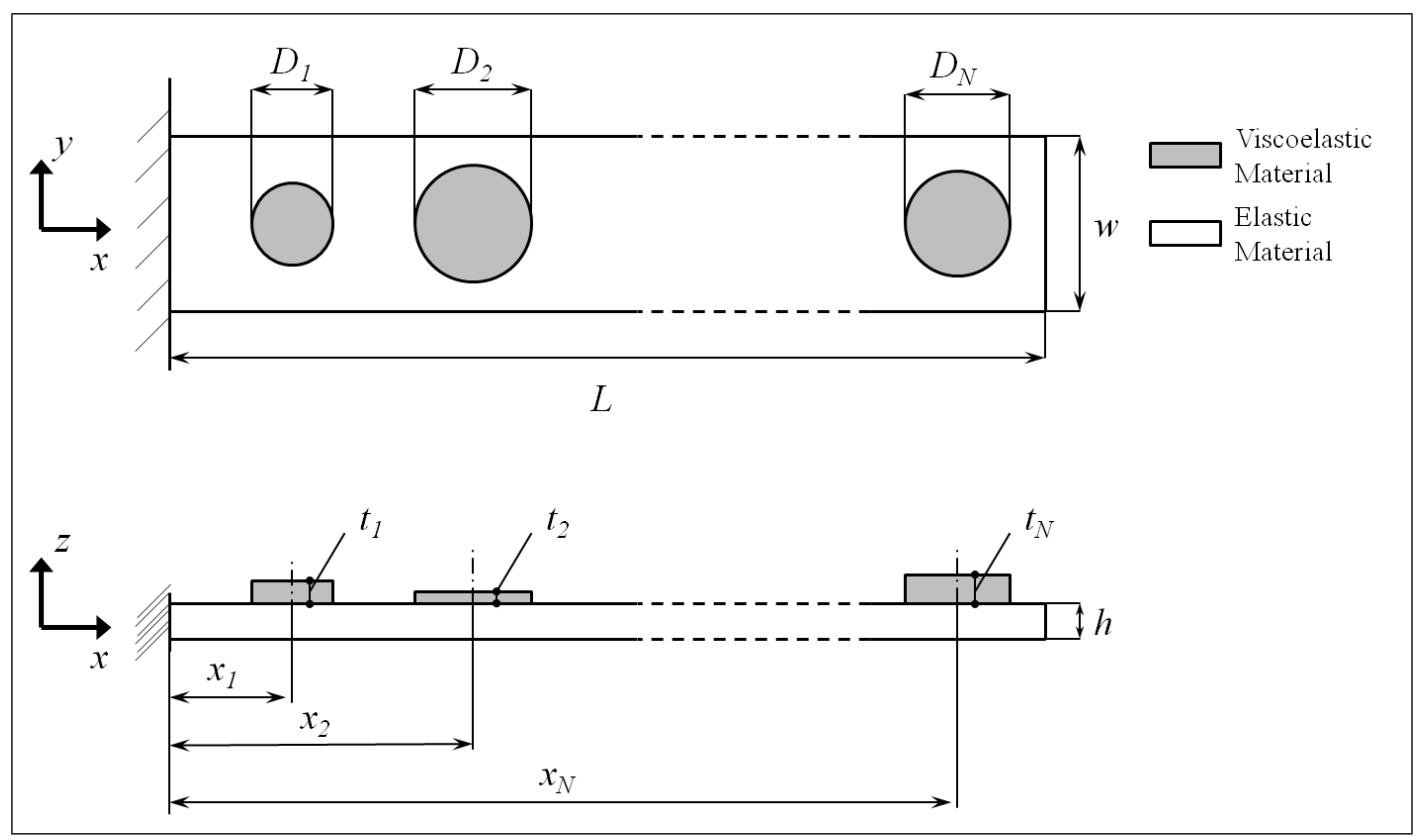

Figure 1. Geometry and design variables of the hybrid structure.

For evident mechanical reasons, we assume that the laminated plate is quasi-homogeneous and fullyorthotropic, with the main orthotropy axis aligned with the $x$ axis of the structure. The definition of a quasi-homogeneous laminate is given in Section 3..

Table 1. Material properties of the glass-epoxy lamina

\begin{tabular}{lrlr}
\hline Technical moduli & \multicolumn{3}{c}{ Polar parameters } \\
\hline Young's modulus $E_{1}[\mathrm{MPa}]$ & 29900 & $T_{0}[\mathrm{MPa}]$ & 5412 \\
Young's modulus $E_{2}[\mathrm{MPa}]$ & 7500 & $T_{1}[\mathrm{MPa}]$ & 5200 \\
Shear modulus $G_{12}[\mathrm{MPa}]$ & 2250 & $R_{0}[\mathrm{MPa}]$ & 3162 \\
Poisson's ratio $\nu_{12}$ & 0.24 & $R_{1}[\mathrm{MPa}]$ & 2841 \\
Density $\rho\left[\mathrm{kg} \mathrm{m}^{-3}\right]$ & 1500 & $\Phi_{0}[\mathrm{deg}]$ & 0 \\
Thickness $t_{p l y}[\mathrm{~mm}]$ & 0.125 & $\Phi_{1}[\mathrm{deg}]$ & 0 \\
\hline
\end{tabular}

In addition, no simplifying assumptions are made on the structure nor on the patches: indeed all patches can be different, in terms of their geometrical parameters. The material used for the viscoelastic patches is a rubber-like material having linear isotropic behaviour. In addition, the material properties depend upon the loading frequency $f$ and they are taken from (Zhang and Chen, 2006). The variation of the complex Young's modulus with the frequency, $E(f)$, is expressed as:

$$
\begin{aligned}
& E(f)=E^{R}(f)+\mathrm{i} E^{I}(f), \\
& \text { with : } \\
& E^{R}(f)=E_{s}{ }^{R}+E_{d}{ }^{R} \log \left(\frac{f}{\tilde{f}}\right), \\
& E^{I}(f)=E_{s}{ }^{I}+E_{d}{ }^{I} \log \left(\frac{f}{\tilde{f}}\right) .
\end{aligned}
$$

The superscripts $R$ and $I$ stand for real and imaginary part, respectively. The subscripts $s$ and $d$ represent the steady-state value and the amplitude of the part that depends upon the frequency for both the real and imaginary part of the Young's modulus, while $\tilde{f}=1 \mathrm{~Hz}$ is a reference value for the frequency.

For our work, we fix the values of parameters in Eq. (1) according to (Zhang and Chen, 2006): $E_{s}{ }^{R}=4.1$ $\mathrm{MPa}$ and $E_{d}^{R}=32.2 \mathrm{MPa}$ are the steady-state value and the amplitude of the real part, while $E_{s}^{I}=-7.7$ 
$\mathrm{MPa}$ and $E_{d}{ }^{I}=43.3 \mathrm{MPa}$ are the corresponding quantities for the imaginary part. The Poisson's ratio and the density are equal to $\nu=0.3$ and $\rho=968.1 \mathrm{~kg} \mathrm{~m}^{-3}$, respectively, whilst the material loss factor $\eta_{v}(f)$ can be determined as:

$$
\eta_{v}(f)=\frac{E^{I}(f)}{E^{R}(f)} .
$$

\subsection{Description of the two-level optimisation strategy}

The design procedure that we propose is inspired by a radical point of view: to develop a mathematically rigorous procedure for the optimum design of modular hybrid structures without the need of simplifying assumptions. Only avoiding the use of a priori assumptions one can hope to obtain the true global optimum for a given problem: this is a key-point in our approach. The design process that we propose is, on one side, not submitted to restrictions introduced artificially to simplify the problem itself, and on the other side completely automatic: the designer does not need to take any preliminary decision, for instance on the number of the patches or of the layers, because the method will do that automatically. In fact, the approach presented in this paper can optimise also the number of modules and, hence, the number of design variables.

The objective of our problem is the maximisation of the damping capabilities of the structure (in terms of the first $N_{f}$ modal loss factors) with constraints on the minimum admissible stiffness and on the maximum admissible weight. Concerning the organisation of the whole optimisation procedure, the problem of designing the damping properties of the hybrid structure is formulated into two distinct problems:

- first-level problem: the aim of this phase is the design of the structure in order to maximise the first $N_{f}$ modal loss factors, satisfying, simultaneously, the constraints on the bending stiffness and on the final weight. At this stage, the laminated plate is considered as an equivalent single layer and its mechanical properties are described by means of the classical stiffness tensor $\mathbf{A}, \mathbf{B}$ and $\mathbf{D}$. We use the polar formalism to represent such tensors and we assume that the laminate is quasi-homogeneous and fully-orthotropic with the main orthotropy axis aligned with the $x$ axis of the structure, see Section 3 .. The output of this step is, hence, the optimal geometry of the structure, i.e. the optimal number $N$ of elastomer patches, their positions over the plate and their geometrical characteristics (diameters and thickness). Further outcomes of this phase are the optimal geometrical and the polar parameters of the laminate, i.e. its total thickness (being the total thickness discretised as a multiple of the elementary layer thickness, the total thickness also indicates the number $n$ of plies composing the laminate) and its anisotropic polar moduli. Thus, this is the step where the true optimal design of the structure is done, in terms of its overall properties;

- second-level problem: the goal of this phase is to find at least one stacking sequence, for the multilayer plate, which is quasi-homogeneous and fully-orthotropic, owning the optimal elastic moduli issued from the first step. At this stage, the design variables are the layer orientations.

In the next Sections, we will give a detailed description of the mathematical formulation of the optimisation problems for both the first and the second steps of the procedure.

\section{Mathematical formulation of the first-level problem}

The overall characteristics of the optimal structure have to be designed during this phase. For the problem at hand, this means that in this phase we have to determine the optimal values of the following parameters:

- the number of viscoelastic circular patches;

- the geometrical properties (position, diameter and thickness) of each patch;

- the thickness, and hence the number of layers, of the laminated plate;

- the elastic properties of the laminate, namely the anisotropic polar moduli of the plate.

Hence, we need to determine the optimal number of modules, i.e. of viscoelastic patches: patches can have different positions and different sizes.

It is worth noting that, for sake of simplicity, we only consider circular patches. Indeed, the aim of this study is to demonstrate the relevance of using patches to damp composite plates and not to discuss their possible geometry. More precisely, circular patches do not need the introduction of the orientation as 
additional design variable for each patch, so this choice minimises the number of geometrical characteristics of the patches.

We can also remark that during this stage of the optimisation procedure, the thickness $h$ of the composite plate must be considered as a discrete optimisation variable, the discretisation step being equal to the thickness of the material layer used for the fabrication of the laminate, see Table 1. Of course, this responds to a technological need and, moreover, this will give us also another important result: the optimal number of layers $n$ to be used during the second-level design phase.

The maximisation of the first $N_{f}$ modal loss factors of the structure must be done satisfying on one side, the mechanical constraints on the bending stiffness and on the weight of the structure, and on the other side the geometric bounds for the elastic moduli along with the geometric constraints on the position of the centre of each patch. Such aspects are described in detail in the following subsections.

\subsection{Geometrical design variables}

Before specifying the mathematical formulation of the first-level problem, we introduce the design variables. These are of two types: geometrical and mechanical. Concerning the geometrical design variables, they are shown in Figure 1 and are:

- the number of patches $N$;

- the position of the centre of each patch $x_{i}, i=1 \ldots N$;

- the diameter $D_{i}$ and the thickness $t_{i}$ of the generic patch, $i=1 \ldots N$;

- the thickness of the laminated plate $h$;

The design variables, their nature and bounds for the first-level problem are detailed in Table 2.

Table 2. Design space for the first-level problem.

\begin{tabular}{lcccc}
\hline Design variable & Type & Lower bound & Upper Bound & Step \\
\hline$N$ & integer & 1 & 10 & 1 \\
$x_{i}[\mathrm{~mm}]$ & discrete & 0.1 & 200.0 & 0.1 \\
$D_{i}[\mathrm{~mm}]$ & discrete & 5.0 & 20.0 & 0.1 \\
$t_{i}[\mathrm{~mm}]$ & discrete & 0.5 & 3.0 & 0.05 \\
$h[\mathrm{~mm}]$ & discrete & 1.0 & 4.0 & 0.125 \\
$R_{0 K}^{A^{*}}[\mathrm{MPa}]$ & continuous & -3162.0 & 3162.0 & - \\
$R_{1}^{A^{*}}[\mathrm{MPa}]$ & continuous & 0.0 & 2841.0 & - \\
\hline
\end{tabular}

It is worth noting that the viscoelastic patches cannot have an arbitrary distribution over the plate, but they must satisfy certain geometrical conditions in order to avoid overlapping between two consecutive patches. Moreover, we have to impose that the patches remain inside the length of the plate. Such constraints can be written as follows:

$$
\left\{\begin{array}{l}
\frac{D_{1}}{2}-x_{1} \leq 0 \\
x_{i-1}+\frac{D_{i-1}+D_{i}}{2}-x_{i} \leq 0,(i=2, \ldots, N) \\
x_{N}+\frac{D_{N}}{2}-L \leq 0
\end{array},\right.
$$

\subsection{Mechanical design variables}

For what concerns the mechanical variables, we use the polar formalism, which gives a representation of any planar tensor by means of a complete set of independent invariants. These invariants are called polar parameters and a great advantage in the design of anisotropic structures is that they are directly linked to the different symmetries of the tensor (Verchery 1979; Vannucci 2005). 
By the polar formalism, the reduced stiffness tensor $\mathbf{Q}$ of an anisotropic layer is expressed as:

$$
\begin{aligned}
& Q_{x x x x}=T_{0}+2 T_{1}+R_{0} \cos 4 \Phi_{0}+4 R_{1} \cos 2 \Phi_{1}, \\
& Q_{x x y y}=-T_{0}+2 T_{1}-R_{0} \cos 4 \Phi_{0}, \\
& Q_{x x x y}=\quad R_{0} \sin 4 \Phi_{0}+2 R_{1} \sin 2 \Phi_{1} \text {, } \\
& Q_{\text {yyyy }}=T_{0}+2 T_{1}+R_{0} \cos 4 \Phi_{0}-4 R_{1} \cos 2 \Phi_{1}, \\
& Q_{\text {yyxy }}=-R_{0} \sin 4 \Phi_{0}+2 R_{1} \sin 2 \Phi_{1} \text {, } \\
& Q_{x x y y}=T_{0} \quad-R_{0} \cos 4 \Phi_{0} .
\end{aligned}
$$

In Eq. (4), $T_{0}, T_{1}, R_{0}, R_{1}, \Phi_{0}-\Phi_{1}$ are the polar tensor invariants. $T_{0}$ and $T_{1}$ are the moduli related to the isotropic part of the tensor, $R_{0}$ and $R_{1}$ are the moduli related to the anisotropic part, while $\Phi_{0}$ and $\Phi_{1}$ are the polar angles. The polar parameters of the material used in this work are given in Table 1. Eq. (4) apply to any other fourth-rank tensor having the elastic tensor symmetries. For more details on the properties of polar parameters, see (Vannucci, 2005).

The Classical Laminated Plate Theory (CLPT) gives the constitutive law of a laminate, linking the internal actions to the deformations of the laminate:

$$
\left\{\begin{array}{l}
\mathbf{N} \\
\mathbf{M}
\end{array}\right\}=\left[\begin{array}{ll}
\mathbf{A} & \mathbf{B} \\
\mathbf{B} & \mathbf{D}
\end{array}\right]\left\{\begin{array}{l}
\varepsilon \\
\kappa
\end{array}\right\} .
$$

In (5), $\mathbf{N}, \mathbf{M}, \varepsilon$ and $\kappa$ are second-rank symmetric plane tensors; $\mathbf{N}$ represents the membrane forces, $\mathbf{M}$ the bending moments, $\varepsilon$ the in-plane strains and $\kappa$ the out-of-plane curvatures. The CLPT gives also the composition laws of these three tensors for a laminate composed of $n$ plies. Such laws depend on the mechanical properties of the layers, on their thickness, orientation and position:

$$
\begin{aligned}
& \mathbf{A}=\sum_{j=1}^{n} \mathbf{Q}_{j}\left(\delta_{j}\right)\left(z_{j}-z_{j-1}\right), \\
& \mathbf{B}=\frac{1}{2} \sum_{j=1}^{n} \mathbf{Q}_{j}\left(\delta_{j}\right)\left(z_{j}^{2}-z_{j-1}^{2}\right), \\
& \mathbf{D}=\frac{1}{3} \sum_{j=1}^{n} \mathbf{Q}_{j}\left(\delta_{j}\right)\left(z_{j}^{3}-z_{j-1}^{3}\right) .
\end{aligned}
$$

The previous laws are independent from the tensor representation and hence apply also to the polar formalism. Some standard passages (see (Vannucci, 2005)) give then the polar parameters of tensors A, B and D:

$$
\begin{aligned}
T_{0}^{A}, T_{0}^{B}, T_{0}^{D} & =\frac{1}{m} \sum_{j=1}^{n} T_{0 j}\left(z_{j}^{m}-z_{j-1}^{m}\right), \\
T_{1}^{A}, T_{1}^{B}, T_{1}^{D} & =\frac{1}{m} \sum_{j=1}^{n} T_{1 j}\left(z_{j}^{m}-z_{j-1}^{m}\right), \\
R_{0}^{A} \mathrm{e}^{4 \mathrm{i} \Phi_{0}^{A}}, R_{0}^{B} \mathrm{e}^{4 \mathrm{i} \Phi_{0}^{B}}, R_{0}^{D} \mathrm{e}^{4 \mathrm{i} \Phi_{0}^{D}} & =\frac{1}{m} \sum_{j=1}^{n} R_{0 j} \mathrm{e}^{4 \mathrm{i}\left(\Phi_{0 j}+\delta_{j}\right)}\left(z_{j}^{m}-z_{j-1}^{m}\right), \\
R_{1}^{A} \mathrm{e}^{2 \mathrm{i} \Phi_{1}^{A}}, R_{1}^{B} \mathrm{e}^{2 \mathrm{i} \Phi_{1}^{B}}, R_{1}^{D} \mathrm{e}^{2 \mathrm{i} \Phi_{1}^{D}} & =\frac{1}{m} \sum_{j=1}^{n} R_{1 j} \mathrm{e}^{2 \mathrm{i}\left(\Phi_{1 j}+\delta_{j}\right)}\left(z_{j}^{m}-z_{j-1}^{m}\right),
\end{aligned}
$$

where superscripts $A, B$ and $D$ stand for the corresponding stiffness tensors. In Eq. (7), $m=1,2,3$ for the extensional, coupling and bending stiffness tensor, respectively. $T_{0 j}, T_{1 j}, R_{0 j}, R_{1 j}, \Phi_{0 j}$ and $\Phi_{1 j}$ are the polar parameters of the reduced stiffness tensor $\mathbf{Q}_{j}\left(\delta_{j}\right)$ of the $j$ th lamina; $\delta_{j}$ is the $j$ th ply orientation measured with respect to the $x$ axis in the global frame of the laminate, while $z_{j}$ and $z_{j-1}$ are the $z$ coordinates of the top and bottom surfaces of the $j$ th layer.

As already said, in this work we use a quasi-homogeneous, orthotropic laminate for the composite plate. Introducing the normalised stiffness tensors, defined as:

$$
\mathbf{A}^{*}=\frac{1}{h_{\mathrm{lam}}} \mathbf{A}, \quad \mathbf{B}^{*}=\frac{2}{h_{\mathrm{lam}}^{2}} \mathbf{B}, \quad \mathbf{D}^{*}=\frac{12}{h_{\mathrm{lam}}^{3}} \mathbf{D},
$$

where $h_{\text {lam }}$ is the overall thickness of the laminate; the conditions for quasi-homogeneity and orthotropy are expressed as: 


$$
\begin{array}{rlr}
\mathbf{B}^{*} & =\mathbf{O} & \text { uncoupling condition, } \\
\mathbf{A}^{*} & =\mathbf{D}^{*} & \text { homogeneity condition, } \\
\Phi_{0}^{A^{*}}-\Phi_{1}^{A^{*}} & =K^{A^{*}} \frac{\pi}{4} & \text { orthotropy condition. }
\end{array}
$$

If the first two conditions of Eq. (9) are satisfied, the laminate is said to be quasi-homogeneous, see (Vannucci and Verchery, 2001). In the third of (9) $\Phi_{0}^{A^{*}}$ and $\Phi_{1}^{A^{*}}$ are the polar angles of the tensor $\mathbf{A}^{*}$. The invariant $K^{A^{*}}$ determines the type of ordinary orthotropy, see (Vannucci 2005; Vannucci 2006), and it can assume only the values 0 or 1 . To be remarked that the second and third of (9) give also bending orthotropy.

The quasi-homogeneity condition ensures that the laminate has identical in-plane and bending behaviours: in this case the design domain of elastic moduli is the same for in-plane and bending, and one single set of polar invariants can describe both elastic behaviours. Quasi-homogeneous laminates are a well-known class of laminates, that are widely used in the design of flexural properties (Vannucci and Verchery, 2001).

A simple result of the polar formalism is that, for the general case of laminates with identical layers, the isotropic moduli of the normalised in-plane and bending behaviour $T_{0}^{A^{*}}, T_{1}^{A^{*}}, T_{0}^{D^{*}}$ and $T_{1}^{D^{*}}$ are identical and equal to those of the elementary layer, $T_{0}$ and $T_{1}$ respectively, see (Vannucci and Verchery, 2001). Thus, the isotropic polar moduli of the laminate are fixed and they only depend on the choice of the material of the elementary layer, so they are no longer design variables.

From the third condition of Eq. (9), we get:

$$
\begin{aligned}
& \cos 4 \Phi_{0}^{A^{*}}=(-1)^{K^{A^{*}}} \cos 4 \Phi_{1}^{A^{*}}, \\
& \sin 4 \Phi_{0}^{A^{*}}=(-1)^{K^{A^{*}}} \sin 4 \Phi_{1}^{A^{*}},
\end{aligned}
$$

relations that can be used in Eq. (4), valid for any fourth-order elasticity-like tensor, so for tensor $\mathbf{A}^{*}$ too. Therefore, introducing the quantity

$$
R_{0 K}^{A^{*}}=(-1)^{K^{A^{*}}} R_{0}^{A^{*}},
$$

thanks to quasi-homogeneity and to the polar formalism, we are reduced to only 3 mechanical design variables for the laminated plate: the polar parameters $R_{0 K}^{A^{*}}, R_{1}^{A^{*}}$, concerning the anisotropic part, and the polar angle $\Phi_{1}^{A^{*}}$, that represents the direction of the orthotropy axis.

Moreover, we have to impose that the main orthotropy axis of the laminate is aligned with the $x$ axis of the structure. This condition can be stated as follows:

$$
\Phi_{1}^{A^{*}}=0
$$

In this first level of the procedure, we must also introduce the geometric and feasibility constraints on the polar parameters, which arise from the combination of the layer orientations and positions within the stack. These constraints assure that the resulting optimal polar parameters can correspond to a feasible laminate, which can be designed during the next second step of the optimisation procedure. Such bounds can be written independently for tensors $\mathbf{A}^{*}$ or $\mathbf{D}^{*}$, and they are of course coincident for the case of quasi-homogeneous laminates. A wide discussion about the expression of geometric bounds using the polar formalism is given in (Vannucci, 2012).

We recall here the expression of such constraints (the quantities without the superscript $A^{*}$ refer to the elementary layer):

$$
\left\{\begin{array}{l}
-R_{0} \leq R_{0 K}^{A^{*}} \leq R_{0}, \\
0 \leq R_{1}^{A^{*}} \leq R_{1}, \\
2\left(\frac{R_{1}^{A^{*}}}{R_{1}}\right)^{2}-1-\frac{R_{0 K}^{A^{*}}}{R_{0}} \leq 0 .
\end{array}\right.
$$

It is worth noting that the first two bounds of Eq. (13) can be easily taken into account as box-constraints, i.e. by properly setting the range of variation of the polar quantities $R_{0 K}^{A^{*}}$ and $R_{1}^{A^{*}}$, see also Table 2 . On the contrary, the third condition of Eq. (13) must be taken into account as a constraint for the structural optimisation problem.

We add a conclusive remark about the quasi-homogeneity assumption: thanks to the first two conditions of Eq. (9) we can impose the orthotropy condition directly on the polar angles of tensor $\mathbf{A}^{*}$, easier to be designed. Strictly speaking, the maximisation of the damping capability of the structure involves only bending and torsional modes and hence it seems natural to design only the out-of-plane stiffness tensor of the laminate, i.e. the tensor $\mathbf{D}^{*}$. Nevertheless, if at the first level of the procedure we do not consider any condition on the 
tensor $\mathbf{A}^{*}$, during the subsequent second step we will not have any criteria to design the membrane behaviour of the laminated plate.

Thus, by imposing the quasi-homogeneity condition we are able, during the first phase, to reduce to only three polar parameters the set of mechanical design variables which completely describe the behaviour of the laminate.

In addition, a second important advantage arises from the quasi-homogeneity condition: only thanks to this assumption we are able to mathematically formalise the set of geometric and feasibility bounds of Eq. (13) that the laminate has to satisfy. In fact, to our best knowledge, only imposing the quasi-homogeneity condition we can write, explicitly and independently for tensors $\mathbf{A}^{*}$ and $\mathbf{D}^{*}$, the exact bounds of Eq. (13) (we recall that for a quasi-homogeneous laminate it is sufficient to write such constraints only for the polar parameters of the tensor $\mathbf{A}^{*}$, because they are automatically satisfied by the corresponding polar quantities of the tensor $\mathbf{D}^{*}$ ). Moreover, if we do not consider the quasi-homogeneity assumption, the geometric and feasibility bounds that the laminate must satisfy cannot be written in a closed form and are no longer independent (for what concerns the membrane and the bending behaviours). For a wide discussion upon the geometric and feasible bounds and the related issues the reader is addressed to (Vannucci, 2012).

Finally, it is worth noting that through the use of the polar formalism, it is possible to optimise directly the homogenised tensors representing the material properties of the composite laminated plate without considering the underlying stacking sequence. The choice of the optimisation variables shown in this paragraph, as well as the introduction of the optimisation constraints of eq. (13), are equivalent to a free-material optimisation of the composite laminated plate: for a more detailed discussion on the application of the polar method to freematerial optimisation of composite structures see (Julien, 2010); concerning more generally the free-material approach of composite laminates, one can see for instance (Bendsøe et al., 1995), (Hörnlein et al., 2001), (Hvejsel et al., 2011).

\subsection{Mathematical statement of the problem}

As said previously, the goal of the global structural optimisation is the maximisation of the damping capabilities of the structure without degrading too much its stiffness nor increasing too much its final weight.

The damping capabilities are estimated in terms of modal loss factors of the structure $\eta_{k},\left(k=1, \ldots, N_{f}\right)$. Since the material properties of the patches depend upon the frequency, the calculation of the loss factors needs an iterative procedure: to this purpose we use the well-known IMSE approach. A wide discussion upon the use of the IMSE approach (and also about the use of similar non-linear techniques) for such kind of problems along with the logical flow of the iterative procedure are detailed in (Montemurro et al., 2012).

The optimisation problem can now be formulated. The maximisation of the first $N_{f}$ modal factors can be expressed as the minimisation of the following objective function:

$$
\Phi=-\sum_{k=1}^{N_{f}} \eta_{k},
$$

that represents the opposite of the sum of the first $N_{f}$ modal loss factors.

Along with the increase of the damping capabilities, the structure must exhibit good properties in terms of stiffness. Indeed, the design of the hybrid structure represents a compromise between its damping capability and the ability of keeping good mechanical properties in terms of stiffness, without increasing too much its weight. This can be done, for instance, introducing constraints on the maximum decrease of the bending stiffness and on the maximum increase of the mass of the plate:

$$
\left\{\begin{array}{l}
\frac{M_{y}^{\mathrm{ref}}-M_{y}}{M_{y}^{\mathrm{ref}}}-\epsilon_{M_{y}} \leq 0, \\
\frac{M-M^{\mathrm{ref}}}{M^{\mathrm{ref}}}-\epsilon_{M} \leq 0 .
\end{array}\right.
$$

In Eq. (15) $M_{y}$ is the bending moment around the $y$ axis measured at the root section of the plate when a unitary displacement $\delta=1 \mathrm{~mm}$ is imposed at the tip. Thus, the bending moment is evaluated as shown in Figure 2 and it represents a measure of the bending stiffness of the structure. In Eq. (15) $M$ is the mass of the whole structure. The superscript "ref" stands for reference value. The reference values of the reaction moment and mass are calculated, before the optimisation process, on a reference undamped structure, i.e. a laminated plate without elastomer patches. The quantities $\epsilon_{M_{y}}$ and $\epsilon_{M}$ are the user-defined tolerances on each constraint. The meaning of the constraints on the reaction moment and on the mass of the hybrid structure 
are the following: the maximum loss in stiffness and the maximum increase in mass of the optimised structure are superiorly bounded by the value of the corresponding tolerances.

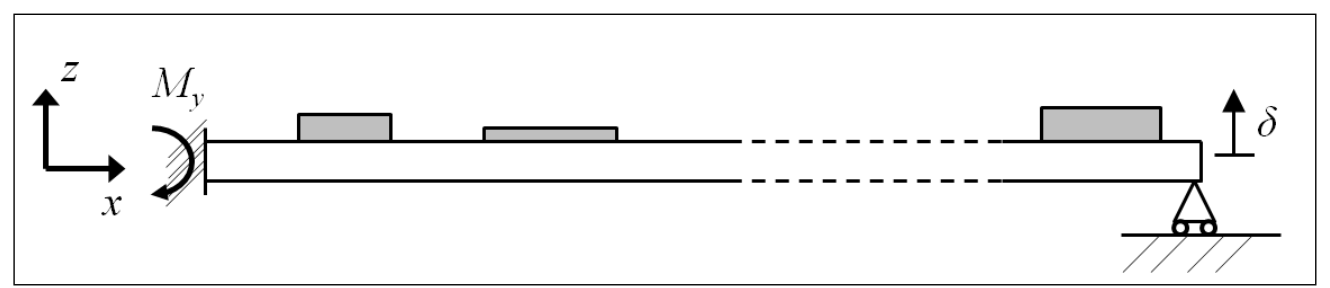

Figure 2. Static test for the evaluation of the bending moment: imposed unitary displacement along $z$ direction.

To state the optimisation problem in a standard form, firstly we reorder the design variables according to the following scheme:

- the vector $\boldsymbol{\xi}$ collects the following design variables, concerning the plate and the number of patches:

$$
\boldsymbol{\xi}=\left\{\begin{array}{c}
\xi_{1}=N \\
\xi_{2}=h \\
\xi_{3}=R_{0 K}^{A^{*}} \\
\xi_{4}=R_{1}^{A^{*}}
\end{array}\right\},
$$

- each one of the vectors $\zeta^{i}$ collects the design variables of the $i$ th viscoelastic patch, $i=1, \ldots, N$ :

$$
\boldsymbol{\zeta}^{i}=\left\{\begin{array}{c}
\zeta_{1}=x_{i} \\
\zeta_{2}=D_{i} \\
\zeta_{3}=t_{i}
\end{array}\right\}
$$

Then, we introduce the following functions:

- the objective function $\Phi$, expressing the damping capabilities of the structure:

$$
\Phi=\Phi\left(\boldsymbol{\xi}, \boldsymbol{\zeta}^{i}\right)
$$

- the functions expressing the two constraints (15) on the bending stiffness and on the weight:

$$
\begin{gathered}
\Psi_{1}\left(\boldsymbol{\xi}, \boldsymbol{\zeta}^{i}\right)=\frac{M_{y}{ }^{\mathrm{ref}}-M_{y}\left(\boldsymbol{\xi}, \boldsymbol{\zeta}^{i}\right)}{M_{y}{ }^{\mathrm{ref}}}-\epsilon_{M_{y}}, \\
\Psi_{2}\left(\boldsymbol{\xi}, \boldsymbol{\zeta}^{i}\right)=\frac{M\left(\boldsymbol{\xi}, \boldsymbol{\zeta}^{i}\right)-M^{\mathrm{ref}}}{M^{\mathrm{ref}}}-\epsilon_{M},
\end{gathered}
$$

- the functions expressing the five geometric constraints (13) on the polar parameters of the plate:

$$
\begin{gathered}
g_{1}\left(\xi_{3}\right)=-\xi_{3}-R_{0}, \\
g_{2}\left(\xi_{3}\right)=\xi_{3}-R_{0}, \\
g_{3}\left(\xi_{4}\right)=-\xi_{4}, \\
g_{4}\left(\xi_{4}\right)=\xi_{4}-R_{1}, \\
g_{5}\left(\xi_{3}, \xi_{4}\right)=2\left(\frac{\xi_{4}}{R_{1}}\right)^{2}-1-\frac{\xi_{3}}{R_{0}},
\end{gathered}
$$

- the functions expressing the $N+1$ geometric constraints (3) on the positions of the patches bonded over the plate:

$$
\begin{gathered}
h_{1}\left(\zeta_{1}^{1}, \zeta_{2}^{1}\right)=\frac{\zeta_{2}^{1}}{2}-\zeta_{1}^{1} \\
h_{i}\left(\zeta_{1}^{i-1}, \zeta_{2}^{i-1}, \zeta_{1}^{i}, \zeta_{2}^{i}\right)=\zeta_{1}^{i-1}+\frac{\zeta_{2}^{i-1}+\zeta_{2}^{i}}{2}-\zeta_{1}^{i},(i=2, \ldots, N), \\
h_{N+1}\left(\zeta_{1}^{N}, \zeta_{2}^{N}\right)=\zeta_{1}^{N}+\frac{\zeta_{2}^{N}}{2}-L .
\end{gathered}
$$


Finally, the problem can be stated in the standard form:

$$
\begin{cases}\min \Phi\left(\boldsymbol{\xi}, \boldsymbol{\zeta}^{1}, \ldots, \boldsymbol{\zeta}^{N}\right), & \\ \text { subject to : } & \\ \Psi_{k}\left(\boldsymbol{\xi}, \boldsymbol{\zeta}^{1}, \ldots, \boldsymbol{\zeta}^{N}\right) \leq 0, & k=1,2, \\ g_{j}(\boldsymbol{\xi}) \leq 0, & j=1, \ldots, 5, \\ h_{i}\left(\boldsymbol{\zeta}^{1}, \ldots, \boldsymbol{\zeta}^{N}\right) \leq 0, & i=1, \ldots, N+1 .\end{cases}
$$

Problem (29) is non-linear, in terms of both geometrical and mechanical variables. Its non-linearity is given not only by the objective function but also by the geometrical constraints on the laminate polar parameters as that in Eq. (25) and by the mechanical constraints on the bending moment and on the weight of the structure, see Eq. (19) and (20).

Finally, the dimension of the design space, i.e. the number of design variables, and the number of constraint equations depend on the number $N$ of patches. In particular, the total number of design variables is $3 N+4$ (there are in fact 3 variables for each patch, 3 variables for the laminated plate and the number of patches, $N$ ), while the total number of constraint equations is $N+8$ : the constraint on the bending moment, the constraint on the weight, 5 constraints for the laminate polar parameters and finally $N+1$ constraints for the position of the patches over the plate, see the second, third and fourth of Eq. (29), respectively. Moreover, though the number of constraints is variable, each constraint due to the addition of a module depends also on the unknowns concerning the others modules, not only on the ones of that module, see again the fourth of Eq. (29) and also Eq. (27).

A final remark about the numerical strategy employed for the solution search: for the resolution of problem (29) we use the new version of the genetic code BIANCA (Montemurro et al., 2012b). In the case of the first-level problem we need the use of the new genetic operators of crossover and mutation between individuals belonging to different species, see (Montemurro et al. 2012a; Montemurro et al. 2012b). In fact, since the number of viscoelastic patches $N$ is included among the design variables, the related optimisation problem is defined over a space having variable dimension (the dimension of such a space is $3 N+4$ ). Mathematically speaking, such a problem corresponds on one side to determine the optimal dimension of the domain (i.e. the optimal number of patches $N$ ) and on the other side to determine the optimal values of the constitutive parameters of the patches for the maximisation of the damping behaviour under all the optimisation constraints defined by the problem (29). In addition, for each individual at each generation, we need to perform a numerical simulation for the evaluation of the objective function as well as of the constraint function on the bending moment. For this reason, the genetic algorithm BIANCA is interfaced with the FE code ANSYS.

Figure 3 shows the genotype of the generic $r$ th individual for the optimisation problem of Eq. (29). This individual has $N_{r}+1$ chromosomes. The first chromosome is composed by 3 genes representing the design variables for the plate, i.e. thickness and polar parameters. Chromosomes from 2 to $N_{r}+1$ contain 3 genes which are the design variables for each patch: position, diameter and thickness. An exception is chromosome 2 that has 4 genes: the fourth additional gene codes the number of modules, i.e. for our problem the number of patches.

Since $N_{r}$ is a variable of our problem, the size of the individual, i.e. the number of chromosomes is variable, which means that different individuals within one population of the genetic algorithm can belong to different species. Symbol $e$ in Figure 3 represents "empty" or inactive genes corresponding to the variable number of constitutive patches/chromosomes for the current individual.

\section{Mathematical formulation of the second-level problem}

The main focus of the second-level phase concerns the design of the laminated plate in terms of its stacking sequence. Of course, this second problem depends upon the results of the first one, because the laminate to be designed must have the optimal elastic properties and thickness issued from the first-level design problem and additionally satisfy the conditions on the elastic symmetries (orthotropy and quasi-homogeneity) introduced in the mathematical formulation of the first-level problem.

In this phase the problem of designing the laminate stack is formulated as an unconstrained minimisation problem. Therefore, the key-point of the second-level problem is the construction of the objective function that drives the search for a quasi-homogeneous, orthotropic laminate, having the optimal elastic polar moduli issued from the first step, i.e. $\widehat{K}^{A^{*}}, \widehat{R}_{0}^{A^{*}}$ and $\widehat{R}_{1}^{A^{*}}$. 


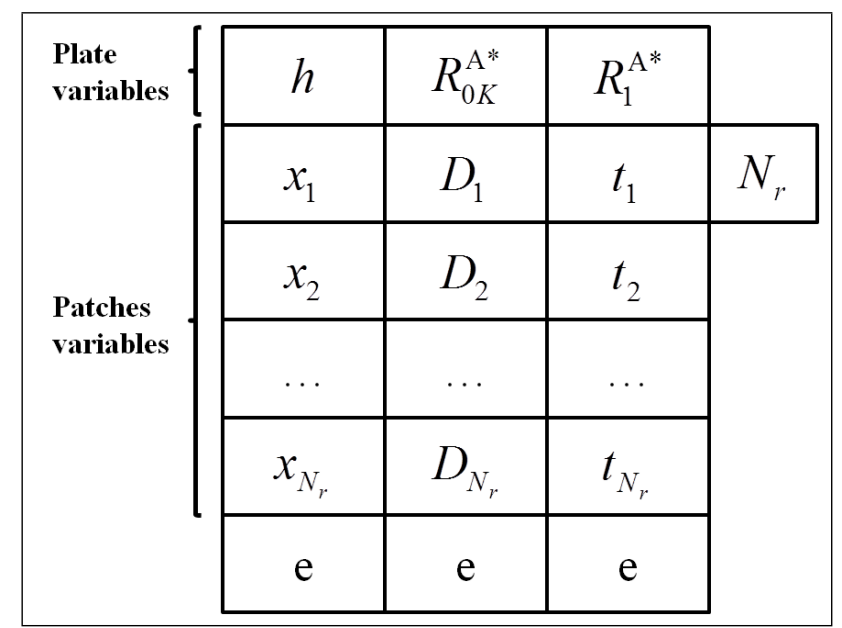

Figure 3. Structure of the individual genotype for the first-level optimisation problem.

All the mathematical details of the second-level problem can be found in (Montemurro et al. 2012a). Here, we recall only the expression of the objective function that assumes the following form:

$$
\begin{aligned}
& \min _{\boldsymbol{\delta}} F(\boldsymbol{\delta})=\sum_{j=1}^{6} f_{j}(\boldsymbol{\delta}) \text { with : } \\
& f_{1}(\boldsymbol{\delta})=\left(\frac{\left\|\mathbf{B}^{*}\right\|}{\|\mathbf{Q}\|}\right)^{2}, f_{2}(\boldsymbol{\delta})=\left(\frac{\|\mathbf{C}\|}{\|\mathbf{Q}\|}\right)^{2}, \\
& f_{3}(\boldsymbol{\delta})=\left(\frac{\Phi_{0}^{A^{*}}-\Phi_{1}^{A^{*}}-\widehat{K}^{A^{*}} \frac{\pi}{4}}{\frac{\pi}{4}}\right)^{2}, f_{4}(\boldsymbol{\delta})=\left(\frac{R_{0}^{A^{*}}-\widehat{R}_{0}^{A^{*}}}{\widehat{R}_{0}^{A^{*}}}\right)^{2}, \\
& f_{5}(\boldsymbol{\delta})=\left(\frac{R_{1}^{A^{*}}-\widehat{R}_{1}^{A^{*}}}{\widehat{R}_{1}^{A^{*}}}\right)^{2}, f_{6}(\boldsymbol{\delta})=\left(\frac{\Phi_{1}^{A^{*}}-\widehat{\Phi}_{1}^{A^{*}}}{\frac{\pi}{4}}\right)^{2} .
\end{aligned}
$$

In $(30), \delta$ is the vector of layer orientations (the true design variables of the second-level problem), while $f_{j}(\boldsymbol{\delta})$ is the $j$ th partial term of the objective function, $j=1, \ldots, 6$. The terms $f_{1}(\boldsymbol{\delta})$ and $f_{2}(\boldsymbol{\delta})$ are related to the quasi-homogeneity conditions, while the third one, $f_{3}(\boldsymbol{\delta})$, is linked to the orthotropy condition, see Eq. (9). The function $f_{3}(\boldsymbol{\delta})$ takes also into account the prescribed value $\widehat{K}^{A^{*}}$ of parameter $K^{A^{*}}$ issued from the first optimisation phase. The terms $f_{4}(\boldsymbol{\delta})$ and $f_{5}(\boldsymbol{\delta})$ correspond to the prescribed optimal values $\widehat{R}_{0}^{A^{*}}$ and $\widehat{R}_{1}^{A^{*}}$ of the polar moduli $R_{0}^{A^{*}}$ and $R_{1}^{A^{*}}$. The term $f_{6}(\boldsymbol{\delta})$ corresponds to the imposed direction of orthotropy of the laminate: $\Phi_{1}^{A^{*}}=\widehat{\Phi}_{1}^{A^{*}}=0$. Finally, $\left\|\mathbf{B}^{*}\right\|$ is the norm of the homogenised coupling tensor, $\|\mathbf{C}\|$ is the norm of the homogeneity tensor, whilst $\|\mathbf{Q}\|$ is the norm of the layer reduced stiffness tensor.

The genetic algorithm BIANCA has also been used in this second step of the procedure. The structure of the genotype of the individual-laminate is shown in Figure 4. The genotype is made of $n$ chromosomes, which correspond to the $n$ constitutive layers of the laminate ( $n$ is determined in the first step) and each chromosome is composed, on its turn, by a single gene which represents the ply orientation.

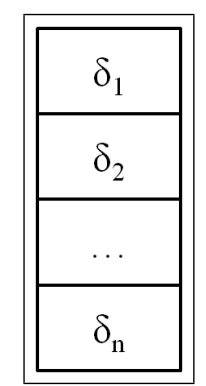

Figure 4. Structure of the individual genotype for the second-level optimisation problem. 
As conclusive remark, it is worth noting that the optimal thickness of the laminated plate is a result of the first-level problem. Since we defined the plate thickness as a discrete variable, which is an integer multiple of the thickness of the elementary ply, then the optimal number of constitutive layers is also known.

\section{Finite element model of the hybrid structure}

The finite element analysis is conducted in order to evaluate the objective and constraint functions for each individual, i.e. for each point in the design space at the current generation. The FE model is built in ANSYS environment, see Figure 5. The need to analyse, within the same generation, different geometrical configurations (plates with different number of patches of different size at different positions), each one corresponding to an individual, requires the creation of an ad-hoc input file for the FE code, that has to be interfaced with BIANCA. Since the number of modules is included among the decision variables, the FE model must be conceived in order to take into account for variable geometry and mesh. Indeed, for each individual at the current generation, depending on the number of chromosomes and, hence, on the number of patches the FE code has to be able to vary in a correct way the number of elements wherein the structure is discretised, thus a correct parametrisation of the model has to be done.

The structure is modelled with a combination of shell and solid elements. In particular, the laminate is modelled using ANSYS SHELL99 elements with 8 nodes and 6 degrees of freedom (DOFs) per node and its mechanical behaviour is described specifying the Cartesian components of tensors $\mathbf{A}^{*}, \mathbf{B}^{*}$ and $\mathbf{D}^{*}$ that can be expressed in terms of the mechanical design variables, i.e. the polar parameters. The viscoelastic patches are modelled using ANSYS SOLID186 elements with 20 nodes and 3 DOFs per node.

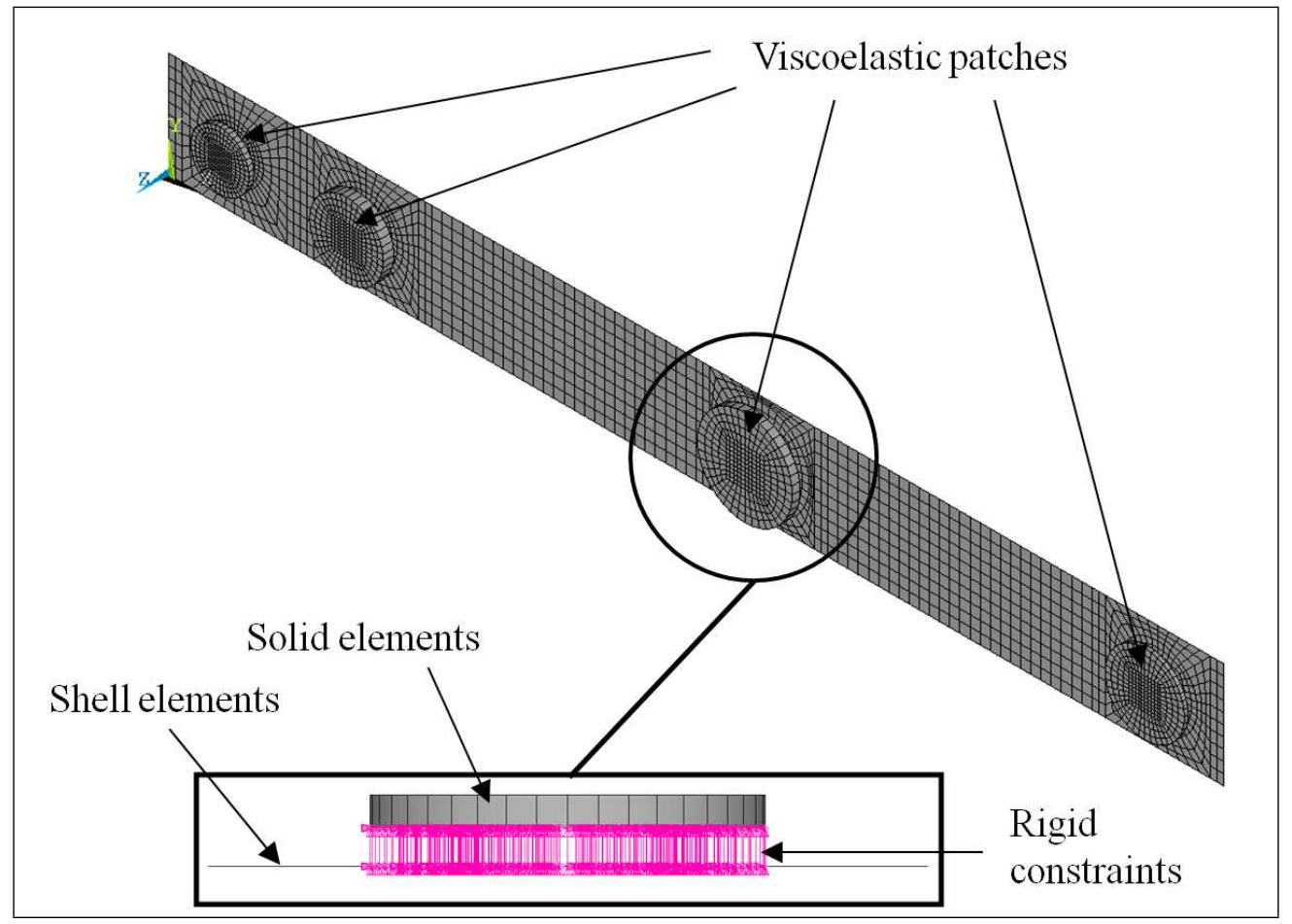

Figure 5. Mesh and rigid constraint equations for the FE model of the structure.

The choice of using solid elements to model the viscoelastic patches is strictly related to the main goal of our optimisation strategy: since we have to estimate the loss factors of the structure, for each natural frequency, we need to build a mathematical model able to describe (with a good level of accuracy and reliability) the mechanical response of the physical system, i.e. the energy dissipation due to the different components of the strain tensor.

It is worth noting that, during the optimisation process, for each individual, we perform two FE calculations: a non-linear modal analysis, according to the logical flow of the IMSE method already presented in (Montemurro et al., 2012), together with a linear static analysis in order to compute the reaction moment $M_{y}$, according to the scheme shown in Figure 2. Concerning the boundary conditions (BCs) of the model, they can vary depending on the considered case-study as we will explain in the next Section. In any case, the extraction of the first $N_{f}=5$ non-rigid modes for the FE model is performed. 
We recall that the number of elements as well as the number of DOFs of the whole structure depends on the number of patches $N$. In particular, the size of the shell elements can vary over the plate surface: in the regions without the viscoelastic patches the sizes of the shell elements are $2 \times 2 \mathrm{~mm}^{2}$, while a local refinement occurs in the regions where the patches are bonded. Moreover, the in-plane size of the solid elements used for the rubber patches exactly matches the one of the refinement realised for the shell elements of the laminate, see Figure 5. In addition, we previously checked that a single quadratic solid element in the thickness of each patch is sufficient to capture in correct way the damping mechanisms linked to the behaviour off the viscoelastic material. Finally, the number of degrees of freedom of the whole model can vary from 18006 to 56688 , depending on the number of patches.

Differently from what is usually done in the literature, we do not need to create fictitious elements to model the "air", which are used to fill the gaps between the elements that constitute the elastomer patches. With a correct parametrisation of the FE model, in terms of geometry and mesh, we can avoid all these difficulties and we are able to consider the exact circular geometry of the patches, without considering polygonal approximation and without introducing additional elements with fictitious properties (the "air") that can alter the results of the analyses.

As conclusive remark, it can be noticed that the compatibility of the displacement field between the patches (modelled with solid elements) and the plate (modelled with shell elements) is realised by means of constraint equations on each corresponding node belonging to contiguous solid and shell elements, see Figure 5. In particular, we specified rigid constraints between the nodes of the middle surface of the laminated plate and the corresponding ones of the bottom surface of the patches. Rigid constraints equations are specified according to the classical scheme implemented within the ANSYS code: the master nodes are those belonging to the middle plane of the composite plate, whilst the slave nodes are those located on the bottom surface of every patch. Through these constraint equations, the displacements of the nodes belonging to the top surface of the plate (in the region wherein the patch is bonded) are equal to those of the nodes belonging to the bottom surface of the patch.

\section{Studied cases and results}

For our optimisation problem we have considered two cases of example. It is worth noting that each case is different from a conceptual point of view. More precisely, in the first example the laminated plate has not to be designed and we must determine the optimal distribution of the rubber patches in order to maximise the damping capability of the system. In addition, this first example is articulated in two sub-cases that differ only for what concerns the boundary conditions (BCs) applied on the plate in order to study their influence on the distribution of the patches.

On the contrary, in the second example we perform the simultaneous design of both the elastic and the viscoelastic parts of the structure, namely the laminate and the patches, in order to show that we can obtain a more effective optimal configuration in terms of damping capabilities of the system. We recall that the design variables, their nature and bounds for the optimisation problem at hand are detailed in Table 2. Now, let us introduce the two examples:

- example 1: as said previously, the goal of this example consists in maximising the first $N_{f}$ modal loss factors of a given plate by simply bonding some rubber patches over the plate. Nevertheless, since the characteristics of the laminated plate (in terms of number of layers and stacking sequence and, hence, in terms of stiffness) are fixed a priori, we do not consider the constraint (19) on the bending stiffness as well as constraints from (21) to (25) on the laminate polar parameters. Of course, being the laminate stack given a priori, we do not need to solve the second-level problem of the optimisation procedure. Therefore, in this example the addition of the damping material is limited only by weight and geometrical requirements: in other words we consider only the constraint (20) on the weight of the plate as well as constraints from (26) to (28) on the positions of the patches for what concerns the mathematical formulation of the optimisation problem.

We remind that the overall number of design variables depends on the number of patches $N$. Thus, for the present case we have $3 N+1$ unknowns: since the laminated plate has not to be designed, the only design variables are the number of patches $N$ along with their constitutive parameters, namely 3 design variables for each patch, i.e. the position $x_{i}$, the diameter $D_{i}$ and the thickness $t_{i}$. In addition, for this first case, the total number of constraints is $N+2: 1$ constraint on the weight of the structure and $N+1$ constraints on the positions of the patches over the plate. In particular, according to the bounds on the number of patches listed in Table 2, the number of design variables varies between 4 and 31 , while the minimum number of constraints is 3 and the maximum one is 12 . 
As said previously, since our aim consists in studying the influence of the BCs of the model on the optimal distribution of the patches over the plate, and, hence, their influence on the damping capabilities of the system, we consider two different sub-cases:

a) case 1.a: the plate is clamped at the root section;

b) case 1.b: the plate is considered simply-supported at both root and tip sections;

- example 2: in this last example we consider the most general case where the optimum design of the system is performed simultaneously for both the elastic and viscoelastic properties of the structure: on one hand we look for the best distribution of the rubber patches and on the other hand we search for the optimal elastic properties of the laminate (in terms of its polar parameters $R_{0 K}^{A^{*}}$ and $R_{1}^{A^{*}}$ and its thickness $h$ ) which maximise the damping capabilities of the system satisfying, at the same time, the requirements on the weight and on the bending stiffness of the structure. Therefore, for this example the first-level problem is formulated in the most general case, according to Eq. (29), considering all constraints. Of course, for this example we need to solve the second-level problem because the laminate stack has to be designed in order to obtain the optimal elastic properties and thickness issued from the first phase of the procedure. In addition, concerning the BCs of the FE model, in this last case the plate is clamped at the root section.

For the present case, the total number of design variables as well as the number of constraint equations depend on the number of patches $N$, as explained in Section 3.: the number of unknowns can vary between 7 and 34, while the number of constraints varies between 9 and 18 .

These test cases are chosen according to the problems often treated in the literature and also according to the will of testing new problems which are more general and complex than the ones already presented in the literature.

Concerning the optimisation problem of Eq. (29), for the first example the laminated plate has a quasihomogeneous, fully-orthotropic unsymmetric stacking sequence made of 18 plies: $\left[-45 / 0 / 45 /-45 / 0 / 45_{3} / 0_{2} /-\right.$ $\left.45_{2} / 0 /-45_{2} / 45_{2} / 0\right]$. The reference value for the mass of the system is evaluated considering the laminate without the rubber patches: the reference mass is $M^{\text {ref }}=0.0135 \mathrm{Kg}$.

Concerning the second example, in order to establish correct reference values for the reaction moment around the $y$ axis and the mass of the hybrid plate, a static analysis is conducted on a reference structure before starting the optimisation process. The undamped reference plate, i.e. a plate without elastomer patches, considered here is made of 32 glass-epoxy laminae (see Table 1 for the material properties) with the following stacking sequence: $\left[0_{2} / 90_{2} / 0_{2} / 45_{2} /-45_{2} / 0_{2} / 90_{2} / 0_{2}\right]_{S}$. Finally, the reference values of the reaction moment and mass of the system for this last example are $M_{y}{ }^{\text {ref }}=-163.0 \mathrm{Nmm}$ and $M^{\mathrm{ref}}=0.0240 \mathrm{Kg}$, respectively.

As conclusive remark, it can be noticed that the user-defined tolerances on the constraints of the problem (29) are set as follows: $\epsilon_{M_{y}}=\epsilon_{M}=0.05$, i.e. the relative maximum loss in stiffness (only for the last example) and the relative maximum increase in mass (for all cases) between the optimised structure and the reference one are limited to $5 \%$.

\subsection{Example 1, case 1.a: clamped quasi-homogeneous orthotropic plate}

As said previously, in this case the laminated plate is clamped at the root section. Since the number of patches is variable and they are not identical, a crossover between species is required and the optimal value of $N$ is an outcome of the search process: the most adapted species automatically issues as a natural result of the Darwinian selection in the genetic algorithm. The genotype of the individual for this case is the one shown in Figure 3 without the first chromosome coding the variables of the laminated plate, i.e. the thickness and the polar parameters.

Concerning the genetic parameters, the population size is $N_{\text {ind }}=40$ and the maximum number of generations is $N_{\text {gen }}=100$. The crossover and mutation probability are $p_{\text {cross }}=0.85$ and $p_{\text {mut }}=1 / N_{\text {ind }}$, while the shift operator and chromosomes number mutation probability are $p_{\text {shift }}=0.5$ and $\left(p_{\text {mut }}\right)_{\text {chrom }}=$ $\left(N_{\max }-N_{\min }\right) / N_{\text {ind }}$, where $N_{\max }$ and $N_{\min }$ are the upper and lower bounds on the number of patches, i.e. the maximum and the minimum number of chromosomes for the generic individual. Selection is performed by the roulette-wheel method, the elitism is active and the Automatic Dynamic Penalisation (ADP) method is used for handling constraints, see (Montemurro et al. 2012b; Montemurro et al. 2013) for more details on the numerical techniques developed within BIANCA and the meaning of the values of different parameters tuning the genetic algorithm BIANCA. 
The best solution found by BIANCA is shown in Table 3. The optimal number of viscoelastic patches for the damping maximisation is 3 . The global constrained minimum was found after 90 generations, see Figure 6 a). Fig $6 \mathrm{~b}$ ) shows the variation of the optimal number of viscoelastic patches along the generations: it can be seen that the best number of patches $N$ varies between 2 and 3 and that the optimal value of $N$ is reached after 80 generations.

In addition, comparing the plots in Figure 6 a) and $6 \mathrm{~b}$ ), one can notice that the convergence towards the best value of the number of modules (here, the number of patches) and that of the objective function are independent. They never occur at the same time, and the optimisation of the number of modules happens always before that of the objective function. Thus we can conclude that the strategy used in BIANCA for evolving simultaneously species and individuals normally leads first to the result of the best species, and then continues to evolve individuals within the best species towards the best individual.

Figure 7 shows the best individual within the population (the best distribution of the patches over the plate for the present case), at the first and at the last generation. We can see that the sizes (diameter and thickness), the positions and the number of patches change during the generations.

Table 3. Best solution found using BIANCA for the optimisation problem (29), case 1.a.

\begin{tabular}{cc}
\hline & Best solution \\
\hline$N$ & 3 \\
$x_{i}[\mathrm{~mm}]$ & $\{12.5,37.5,62.5\}$ \\
$D_{i}[\mathrm{~mm}]$ & $\{17.7,10.1,15.7\}$ \\
$t_{i}[\mathrm{~mm}]$ & $\{1.46,0.66,1.47\}$ \\
$\eta_{1}$ & 0.01060 \\
$\eta_{2}$ & 0.00846 \\
$\eta_{3}$ & 0.00129 \\
$\eta_{4}$ & 0.01061 \\
$\eta_{5}$ & 0.01440 \\
$f_{1}[\mathrm{~Hz}]$ & 29.43 \\
$f_{2}[\mathrm{~Hz}]$ & 181.85 \\
$f_{3}[\mathrm{~Hz}]$ & 256.09 \\
$f_{4}[\mathrm{~Hz}]$ & 503.74 \\
$f_{5}[\mathrm{~Hz}]$ & 558.53 \\
$M[\mathrm{Kg}]$ & $0.014174(+4.99 \%)$ \\
$\Phi$ & -0.04538 \\
\hline
\end{tabular}

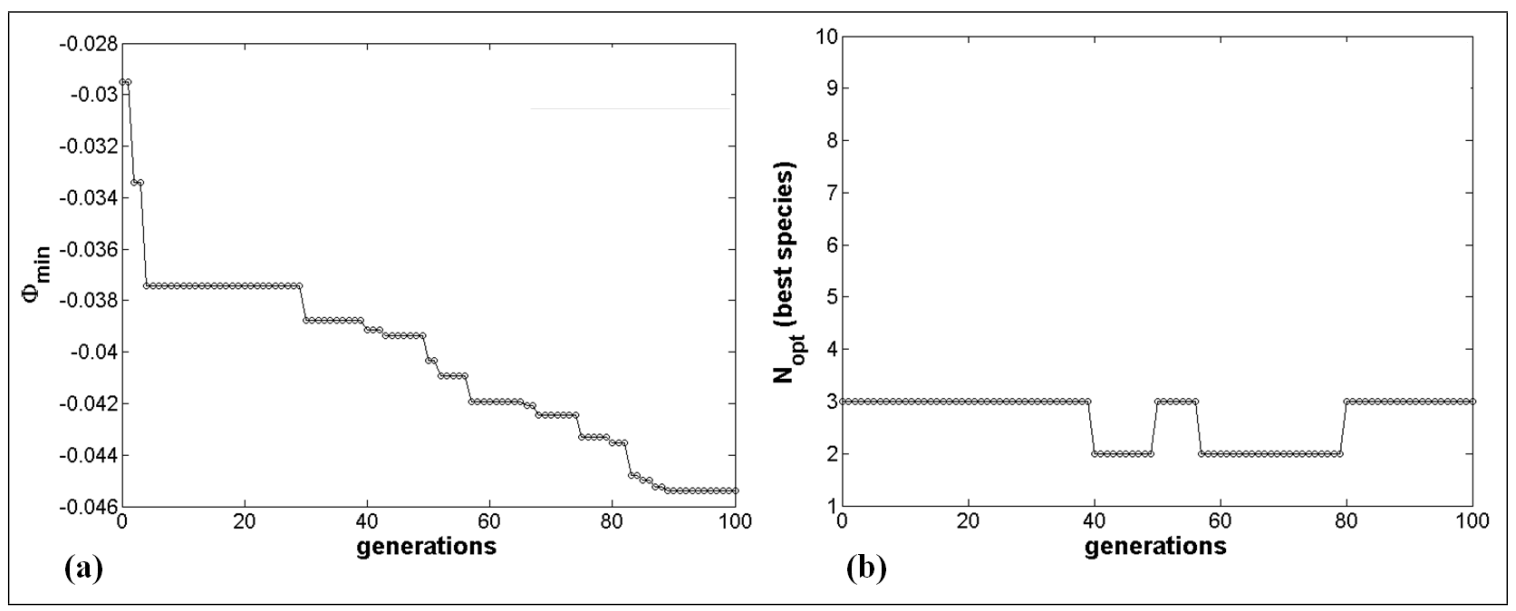

Figure 6. (a) Best values of the objective function and (b) optimal number of patches along generations for problem (29), case 1.a. 


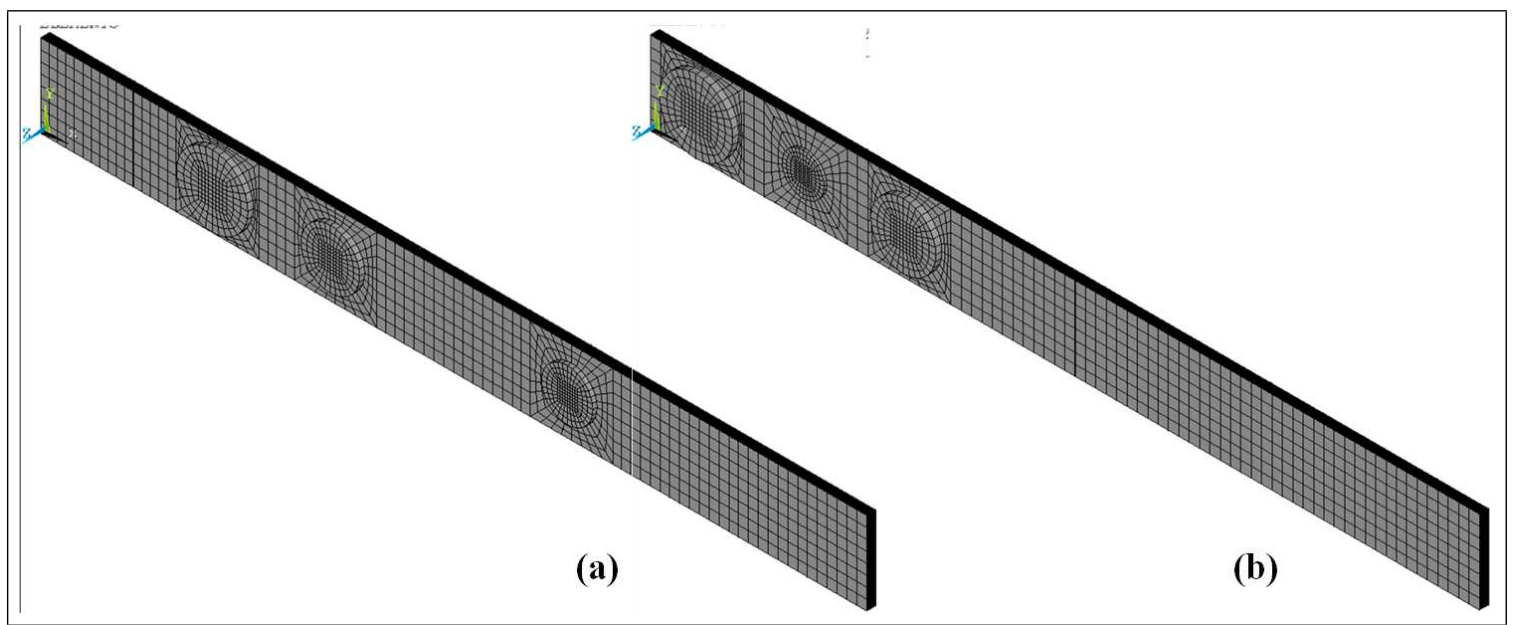

Figure 7. Best distribution of viscoelastic patches at the (a) initial and (b) final generation (optimum) for problem (29), case 1.a.

\subsection{Example 1, case 1.b: simply supported quasi-homogeneous orthotropic plate}

In this second case the laminate is simply-supported at its ends. The aim of such an example is to study how the distribution of the viscoelastic material changes when we consider different BCs for the model. The genotype of the individual and the genetic parameters are exactly the same as the previous case.

The best solution found by BIANCA is shown in Table 4. For this case, the optimal number of viscoelastic patches for the damping maximisation is 2. Figure 8 a) and 8 b) show the variation of the best solution and that of the best species along the generations, respectively. We can see that the global constrained minimum was found after 73 generations, while the number of viscoelastic patches $N$ converges to its optimal value after 13 generations. Again, the convergence towards the best value of $N$ and that of the objective function are independent, and the convergence towards the best species is faster than the convergence towards the best individual.

Figure 9 shows the best individual within the population (the best distribution of the patches over the plate for the present case), at the first and at the last generation. Again, according also with Figure $8 \mathrm{~b}$ ), it can be noticed that the geometry (in terms of diameter and thickness), the positions and the number of patches change during the generations.

Table 4. Best solution found using BIANCA for the optimisation problem (29), case 1.b.

\begin{tabular}{cc}
\hline & Best solution \\
\hline$N$ & 2 \\
$x_{i}[\mathrm{~mm}]$ & $\{25.0,175.0\}$ \\
$D_{i}[\mathrm{~mm}]$ & $\{17.8,16.8\}$ \\
$t_{i}[\mathrm{~mm}]$ & $\{1.50,1.45\}$ \\
$\eta_{1}$ & 0.00196 \\
$\eta_{2}$ & 0.00854 \\
$\eta_{3}$ & 0.01563 \\
$\eta_{4}$ & 0.01620 \\
$\eta_{5}$ & 0.01808 \\
$f_{1}[\mathrm{~Hz}]$ & 80.78 \\
$f_{2}[\mathrm{~Hz}]$ & 319.34 \\
$f_{3}[\mathrm{~Hz}]$ & 712.52 \\
$f_{4}[\mathrm{~Hz}]$ & 1078.07 \\
$f_{5}[\mathrm{~Hz}]$ & 1268.08 \\
$M[\mathrm{Kg}]$ & $0.014172(+4.98 \%)$ \\
$\Phi$ & -0.06041 \\
\hline
\end{tabular}




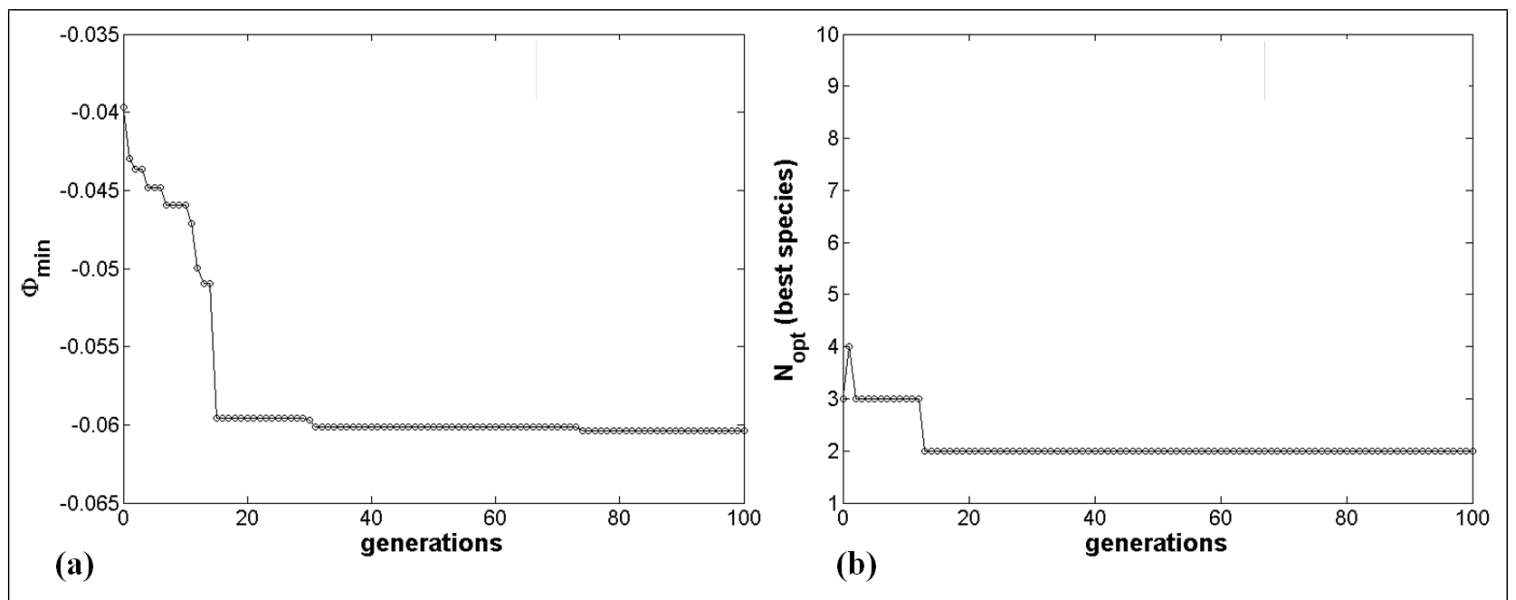

Figure 8. (a) Best values of the objective function and (b) optimal number of patches along generations for problem (29), case 1.b.

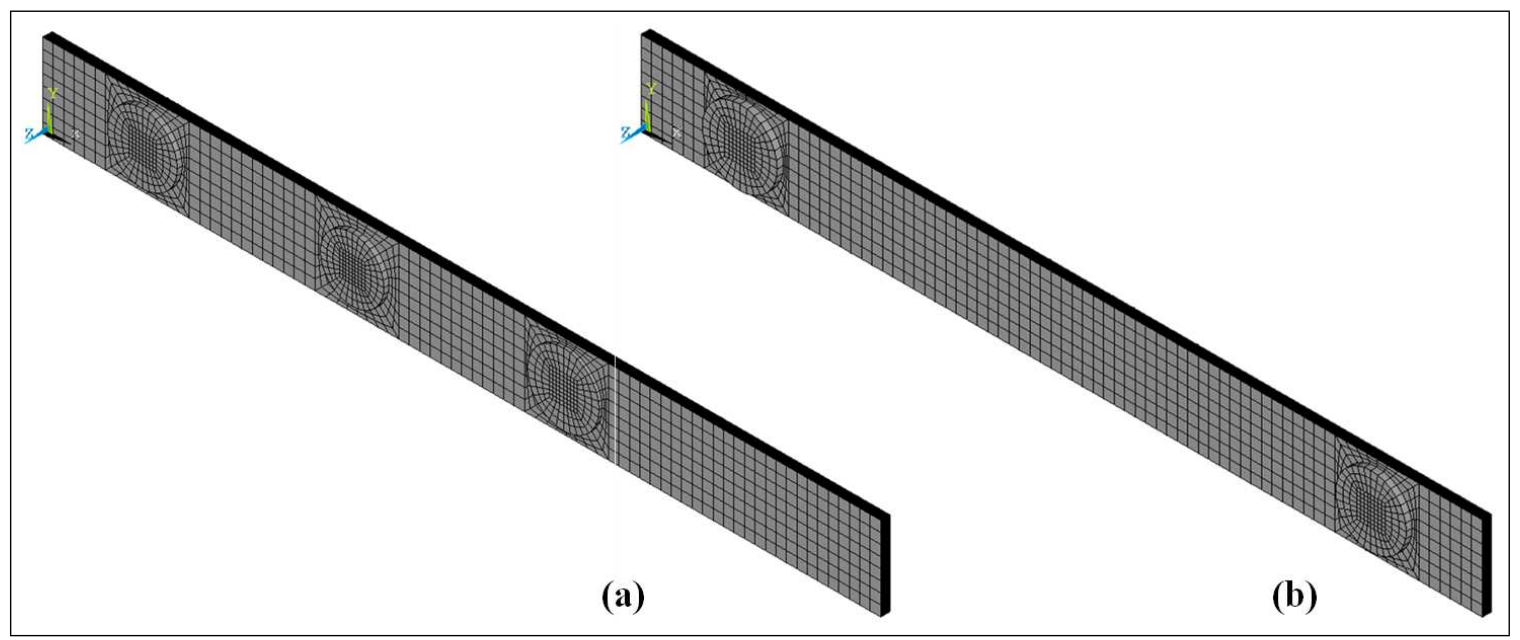

Figure 9. Best distribution of viscoelastic patches at the (a) initial and (b) final generation (optimum) for problem (29), case 1.b.

\subsection{Example 2: general case}

This is the most general case where the design of both the elastic and viscoelastic parts of the structure is realised simultaneously in order to maximise its damping capabilities. We recall that in this last example also the constitutive parameters of the laminate, i.e. its total thickness and the anisotropic polar parameters, are included among the design variables. The genotype of the individual is the one discussed in Section 3 . and shown in Figure 3.

Due to the greater complexity of the optimisation process in the present case, the population size and the maximum number of generations are increased up to $N_{\text {ind }}=60$ and $N_{\text {gen }}=150$, respectively. For the rest, the genetic parameters are strictly the same already used in the previous examples.

The best solution found by BIANCA is shown in Table 5. The optimal number of viscoelastic patches for the damping maximisation for this last case is 7 . The global constrained minimum was found after 111 generations, see Figure $10 \mathrm{a}$ ). Fig $10 \mathrm{~b}$ ) shows the variation of the optimal number of viscoelastic patches along the generations: at the first generation the best species shows 3 patches bonded over the plate. Then, one can see that the best number of patches $N$ varies between 5 and 7 and that the optimal value of $N$ is reached after 42 generations. Thus, as in the previous examples, the convergence towards the best value of $N$ and that of the objective function are independent, and the convergence towards the best species is faster than the convergence towards the best individual.

Figure 11 shows the optimal distribution of the patches over the plate for the present case, at the first and at the last generation. Once again, the sizes (diameter and thickness), the positions and the number of patches change during the generations. 
Table 5. Best solution found using BIANCA for the optimisation problem (29), case 2.

\begin{tabular}{cc}
\hline \multicolumn{3}{c}{ Best solution } \\
\hline$N$ & 7 \\
$x_{i}[\mathrm{~mm}]$ & $\{12.5,37.5,62.5,87.5,112.5,137.5,162.5\}$ \\
$D_{i}[\mathrm{~mm}]$ & $\{17.0,16.3,16.3,16.3,16.1,17.6,17.7\}$ \\
$t_{i}[\mathrm{~mm}]$ & $\{3.00,3.00,3.00,3.00,3.00,2.20,2.50\}$ \\
$h[\mathrm{~mm}]$ & 3.5 \\
$R_{0 K}^{A^{*}}[\mathrm{MPa}]$ & 3131.09 \\
$R_{1}^{A^{*}}[\mathrm{MPa}]$ & 2768.79 \\
$\eta_{1}$ & 0.01350 \\
$\eta_{2}$ & 0.00298 \\
$\eta_{3}$ & 0.01652 \\
$\eta_{4}$ & 0.09163 \\
$\eta_{5}$ & 0.01815 \\
$f_{1}[\mathrm{~Hz}]$ & 59.54 \\
$f_{2}[\mathrm{~Hz}]$ & 327.51 \\
$f_{3}[\mathrm{~Hz}]$ & 367.71 \\
$f_{4}[\mathrm{~Hz}]$ & 557.45 \\
$f_{5}[\mathrm{~Hz}]$ & 1022.21 \\
$M[\mathrm{Kg}]$ & $0.02518(+4.93 \%)$ \\
$M_{y}[\mathrm{Nmm}]$ & $-158.87(-2.53 \%)$ \\
$\Phi$ & -0.14278 \\
\hline
\end{tabular}

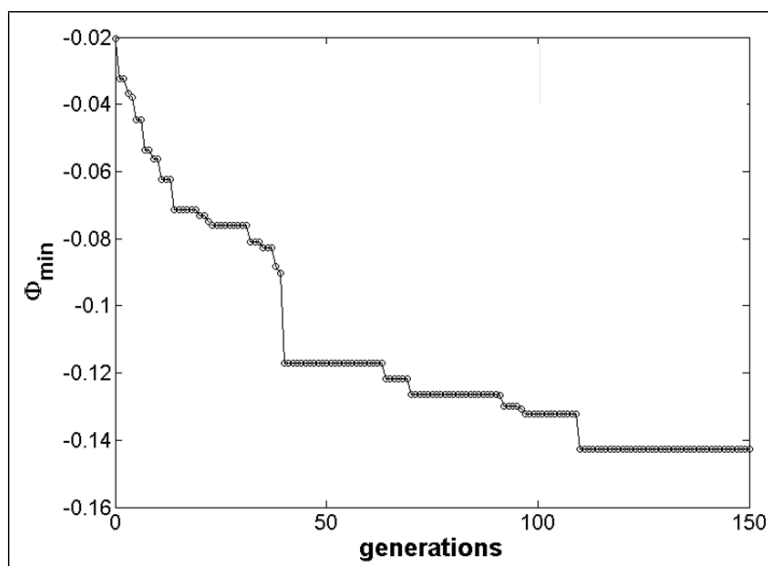

(a)

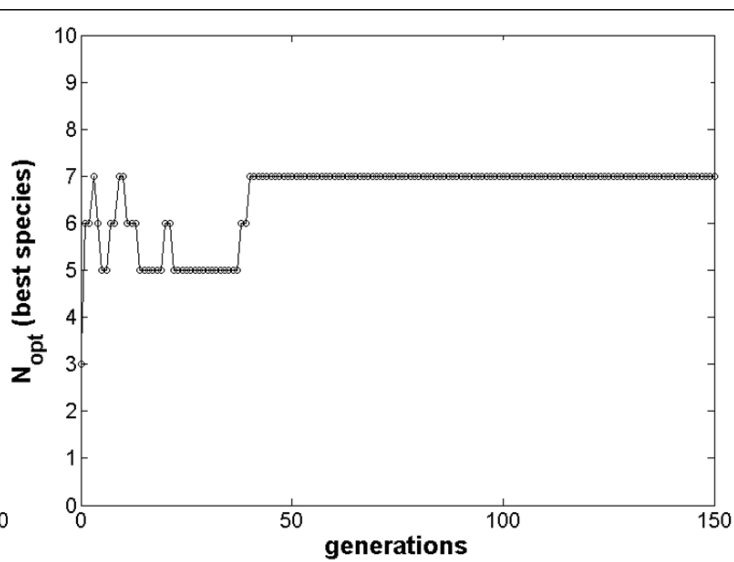

(b)

Figure 10. (a) Best values of the objective function and (b) optimal number of patches along generations for problem (29), case 2 .

The first five non-rigid modes for the optimal configuration of the whole structure are shown in Figure 12. It can be noticed that we have different kinds of modes: the first, the third and the fifth mode are bending modes in the $x-z$ plane of the plate, whilst the second one is a bending mode in the $x-y$ plane of the plate and finally the fourth mode is a torsional mode around the $x$ axis. Of course, the optimal distribution of the viscoealstic material, i.e. the distribution of the rubber patches over the plate, along with the elastic properties of the composite plate are influenced by all the modes and the related damping mechanisms.

Considering that the value of the ply thickness is $0.125 \mathrm{~mm}$, from Table 5 we can notice that the laminated plate is made of 28 plies (the total optimum thickness of the laminate is $h=3.5 \mathrm{~mm}$ ) and has the orthotropy with $\widehat{K}^{A^{*}}=0$, because the value of the polar quantity $R_{0 K}^{A^{*}}$ is positive.

Concerning the second-level problem, the design variables are the layers orientations, which can vary between $-90^{\circ}$ and $90^{\circ}$ with a step of $1^{\circ}$. The population size is set to $N_{\text {ind }}=500$ and the maximum number of generations to $N_{\text {gen }}=500$. The crossover and mutation probability are still $p_{\text {cross }}=0.85$ and 


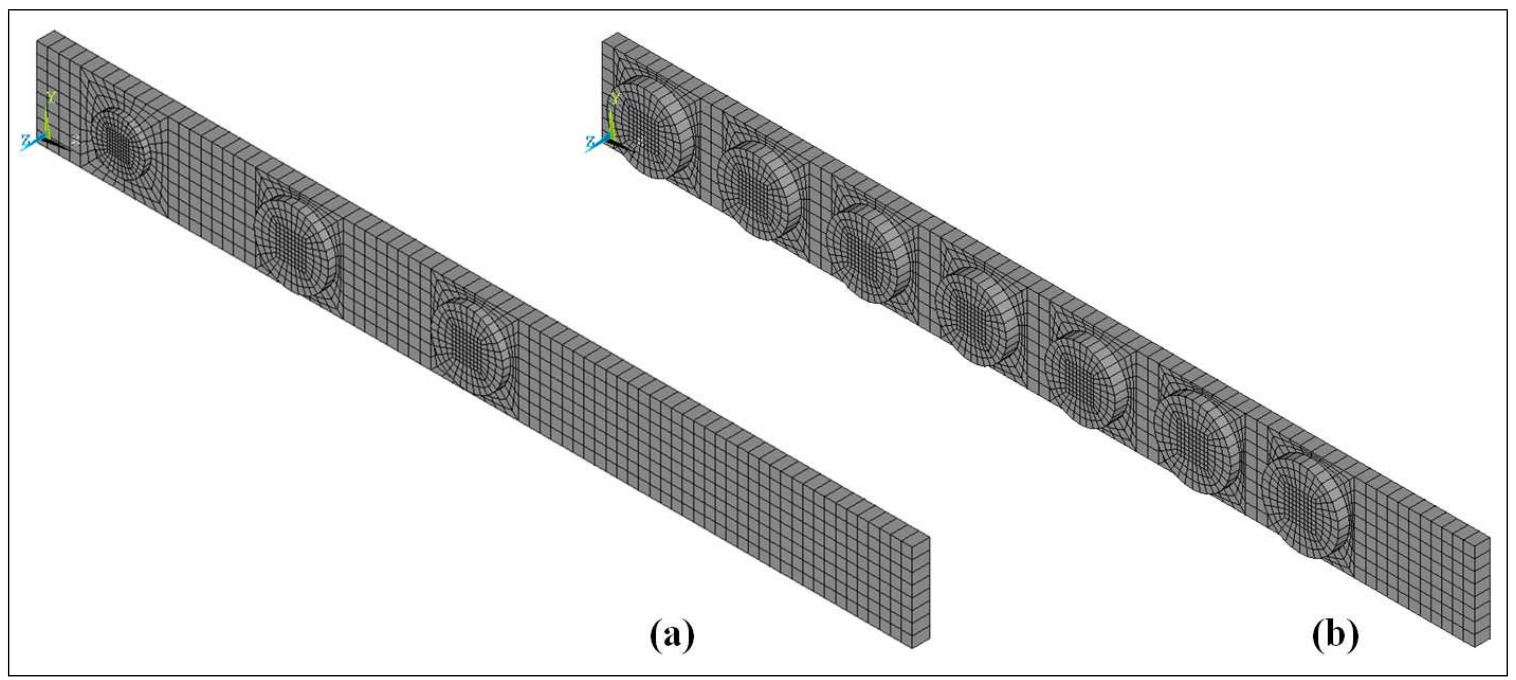

Figure 11. Best distribution of viscoelastic patches at the (a) initial and (b) final generation (optimum) for problem (29), case 2.

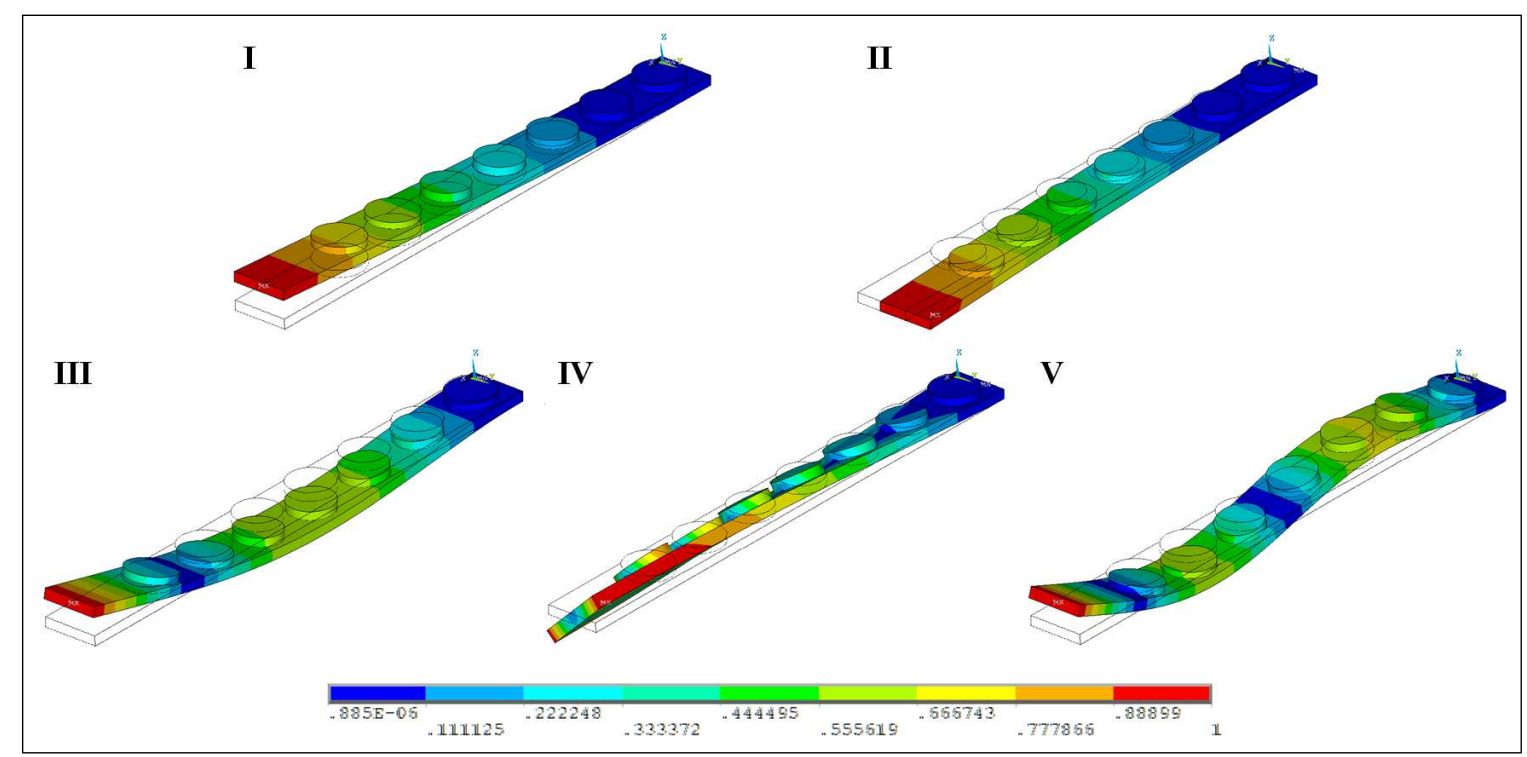

Figure 12. First five non-rigid modal deformed shapes of the whole structure for the optimal configuration, case 2 .

$p_{\text {mut }}=1 / N_{\text {ind }}$, respectively. Selection is performed by the roulette-wheel operator and the elitism is active. Moreover, always concerning the second-level problem, as in each numerical technique, the quality of solutions found by BIANCA can be estimated on the basis of a numerical tolerance, that is the residual. For a discussion on the importance of the numerical residual in problems of this type, the reader is addressed to (Vincenti et al., 2012 (In press)). It is worth noting that, being $F(\boldsymbol{\delta})$ a non-dimensional function, the residual of the solution is a non-dimensional quantity too.

Table 6 shows the best stacking sequence found using BIANCA for the second-level problem. The residual in the last column is the value of the global objective function $F(\boldsymbol{\delta})$ for the solution indicated aside (we remind that exact solutions correspond to the zeroes of the objective function). Figure 13 a) shows the first component of the homogenised stiffness tensors of the laminate, i.e. $\mathbf{A}^{*}, \mathbf{B}^{*}$ and $\mathbf{D}^{*}$ : the solid line refers to the extension tensor, the dashed one to the bending tensor, while the dash-dotted one is linked to the coupling stiffness tensor. We can see that the laminate is uncoupled (the dash-dotted curve is reduced to a small point in the center of the plot, because $B_{11}^{*}$ is "technically" null), homogeneous (the solid and dashed curves are almost coincident) and orthotropic (there are two orthogonal axes of symmetry in the plane). Moreover, the main orthotropy axis is aligned with the $x$ axis of the structure, in fact it is oriented at $0^{\circ}$. Similar considerations could be done for the other components of these tensors, not shown in Figure 13 a) for 
the sake of brevity.

Figure $13 \mathrm{~b}$ ) shows the variation of the best solution during iterations: the best solution is found after 470 generations.

Table 6. Case 2: best stacking sequences for the optimal solution.

\begin{tabular}{clc}
\hline N. of plies & Stacking sequence $\left(^{\circ}\right)$ & Residual \\
\hline \multirow{2}{*}{28} & {$[0 /-1 / 0 /-2 / 0 / 0 / 17 /-1 / 2 /-4 / 0 /-2 /-1 /-6 /$} & $1.3 \times 10^{-3}$ \\
& $-16 /-1 /-1 / 17 / 2 / 17 /-4 /-3 /-1 / 0 / 0 /-2 / 0 / 0]$ & \\
\hline
\end{tabular}

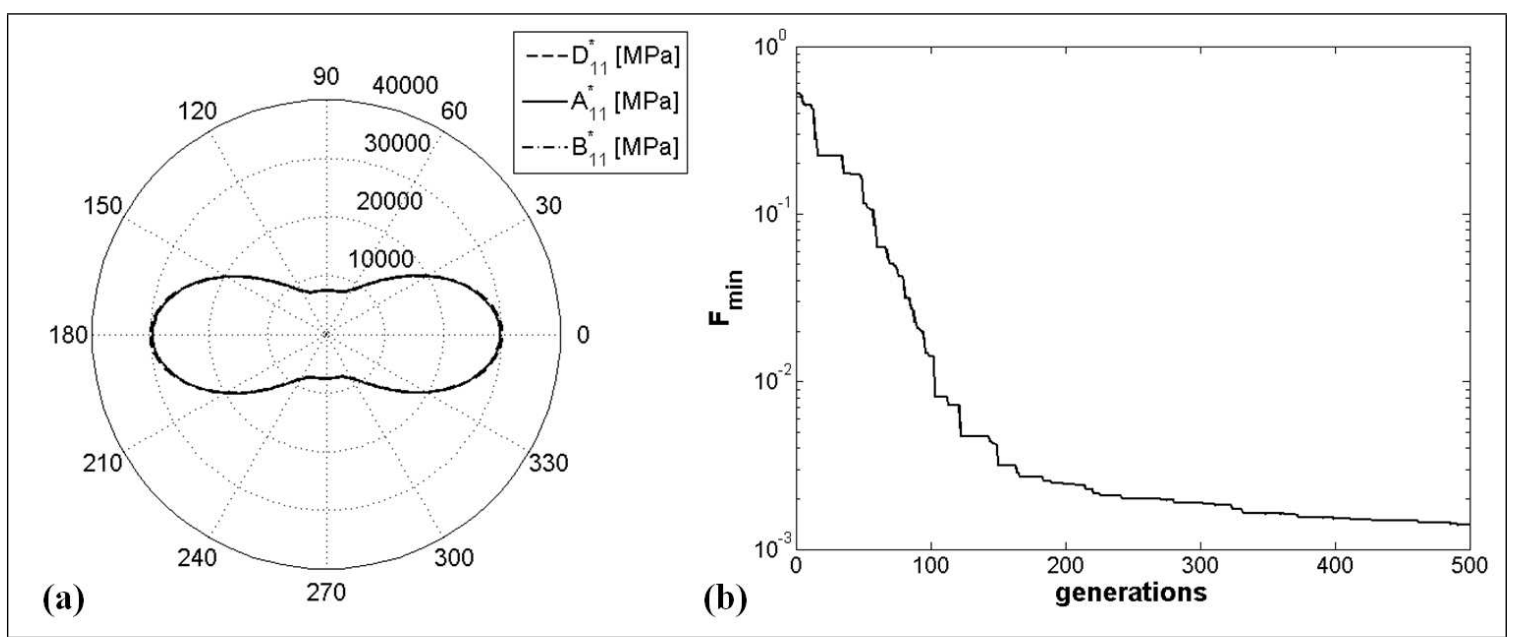

Figure 13. (a) First component of the homogenised stiffness tensors of the laminate and (b) best values of the objective function during iterations, case 2 .

\subsection{Discussion of the results}

Concerning the influence of the BCs of the model on the optimal distribution of the viscoelastic patches we can immediately see this effect by considering the results of the cases 1.a and 1.b of the first example. In case 1.a the patches are placed near to the clamped root section of the plate, i.e. in the region where the strains are higher and, consequently, where the damping phenomenon linked to those strain components is stronger. The same considerations can be done also for the distribution of the patches for case 1.b: in this case the distribution is almost symmetric for what concerns the values of the diameters, thickness and positions, see Table 4, coherently with the symmetric boundary conditions. As in case 1.a, also in this second case the patches are placed in the regions where the plate is simply supported, i.e. where the strains are higher.

Figure $14 \mathrm{a}$ ) and b) show the maximum strain components in the rubber patches for what concerns cases 1.a and 1.b. It can be noticed that, when we consider bending modes in the $x-z$ plane (i.e. modes n. 1,3 and 4 for case 1.a and modes n. 1, 3 and 5 for case 1.b) despite all the strain components are involved into the damping phenomenon, the major contribution is due to the axial strain $\varepsilon_{x x}$ and to the shear strain throughthe-thickness $\varepsilon_{x z}$. The same thing happens when we look at the bending modes in the $x-y$ plane, i.e. mode n. 2 for both cases 1.a and 1.b. On the contrary, when we consider torsional modes, i.e. mode n. 5 for case 1.a and mode n. 4 for case 1.b, the major contribution to the damping mechanism of the structure is associated to the in-plane shear strains $\varepsilon_{x y}$ and, secondly, to the shear deformations $\varepsilon_{x z}$ and $\varepsilon_{y z}$, being the effect of the longitudinal strains negligible for the torsional mode. It is worth noting that, differently from what is usually done in the literature where only bending modes are considered when studying the damping capabilities of viscoelastic materials, all the different modes, i.e. bending and torsional ones, are taken into account into the proposed optimisation procedure because they have the same influence on the damping response of the structure in terms of the values of the modal loss factors.

Concerning the second example, the simultaneous optimisation of the viscoelastic and elastic properties of the structure allows to find better damping capabilities when compared to the first two cases. Only by considering the overall design of the structure one can hope to find a real global optimum configuration: for this last case, in fact, the damping capability of the structure (and, hence, the values of the modal loss factors) 


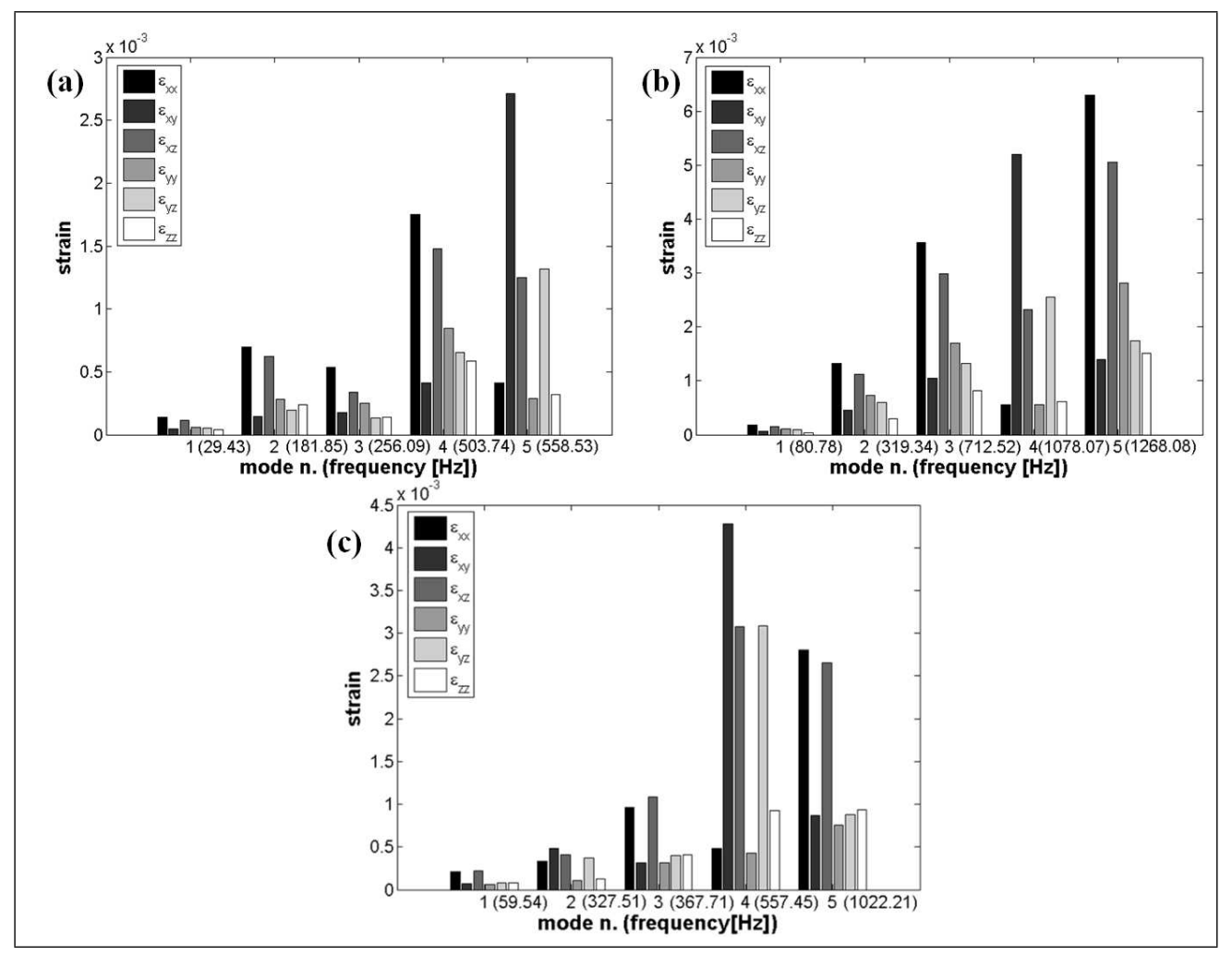

Figure 14. Maximum strain components in the viscoelastic patches for the optimised plate, (a) case 1.a, (b) case $1 . b$ and (c) case 2 .

is practically doubled with respect to that of the first example. Thus, this last example demonstrates that the design of the "damping system" (the viscoelastic patches) must be integrated into the global design of the whole structure.

Figure $14 \mathrm{c}$ ) shows the maximum strain components in the rubber patches for what concerns the example 2. The considerations made for the two sub-cases of the first example can be applied also to this second example. Nevertheless, we can notice that the major contribution to the damping phenomenon of this last case is due to the torsional mode, i.e. mode n. 4 in Figure $14 \mathrm{c}$ ): the value of the modal loss factor associated to that mode is nine times the value of the others loss factors, see Table 5 . This means that the torsional mode of the plate has a strong effect on the final distribution of the patches over the plate, in terms of diameters, thickness and positions.

As a conclusive remark, for what concerns the second example, it can be noticed that, differently from what is usually done in the literature, we do not make any simplifying assumption on the laminate stacking sequence which is completely free, neither we use standard orientation angles for the elementary plies (like for instance $0^{\circ}, \pm 45^{\circ}$ and $90^{\circ}$ ).

All the previous circumstances lead us to find a non-standard optimal solution which represents a real global optimum (in terms of the overall properties of the system) for the problem at hand.

\section{Conclusions}

The optimisation procedure presented in this work is characterised by several points that make it an innovative, effective and general method for the design of hybrid modular structures. Our motivation was to create a general procedure for the optimisation of modular systems, with the number of modules that belongs to the set of the design variables and without using special assumptions. The numerical method is, however, a fundamental part of the procedure, because it is thanks to an appropriate numerical tool that we can simultaneously optimise the number of modules and their characteristics. We briefly recall the features of the procedure:

- no simplifying assumptions nor standard rules are used to design the laminated plate (this allows us to look for a true global minimum, hard to be obtained otherwise); 
- the procedure is composed by two distinct but linked non-linear minimisation problems: the first one is a constrained problem that uses a free-material approach to the design of the geometric and material properties of the modular composite structure via the polar representation of $2 \mathrm{D}$ elasticity; the second step is an unconstrained problem formulated to design a laminate able to realise the overall optimal mechanical properties designed in the first step;

- quasi-homogeneous sequences are used; this allows us to write exact geometric bounds, valid for both the extension and bending behaviour and to reduce the number of mechanical design variables in the first step;

- bending orthotropy is really fulfilled, its type specified and the orthotropy direction directly managed, without using special sequences or orientations;

- the number of modules, i.e. the number of viscoelastic patches as well as the number of layers of the laminated plate, is directly optimised by the procedure. The optimisation of the number of constitutive modules is possible thanks to a genetic approach which is able to select not only individuals, but also species: in practice, the algorithm determines automatically the optimal number of design variables;

- the mechanical characteristics of the laminated plate are represented by the polar formalism, that gives several advantages, namely to explicit elastic symmetries, elastic and geometric bounds, and to eliminate from the procedure redundant mechanical properties;

- the numerical computations are carried out by a special GA, the code BIANCA, able to cross simultaneously species and individuals, to handle continuous and discrete valued variables during the same iterations and to effectively handle the constraints imposed to the problem;

- for the solution of the first-level problem, the code BIANCA is interfaced with a FE code, in order to numerically compute some mechanical quantities, namely the modal loss factors of the structure and the bending moment around the $y$ axis;

- the mathematical formulation of the second step problem allows us to take into account all the possible combinations of requirements on elastic properties; it is stated as the unconstrained minimisation of a positive semi-definite function; being the minima of this function equal to zero, it is possible to know when a global minimum is attained.

To our best knowledge, this is the first time that the problem of maximising the damping capabilities is formulated considering a discontinuous aperiodic distribution of viscoealstic material, namely by considering elastomer patches bonded over the structure which share the same form but, at the same time, they can be characterised by different values of their constitutive geometrical parameters, i.e. diameters, thickness and positions. Moreover, this is also the first time that the optimisation problem is stated considering, in the form of constraint functions, the geometrical requirement on the position of the patches bonded over the plate. Thus, the main key points of our strategy consist in determining what are: a) the best number of rubber patches, b) their best dimensions and positions over the structure as well as c) the best values of the thickness and of the polar parameters of the laminated plate which play an important role in the determination of the damping capability of the system.

The use of an evolutionary strategy along with the fact that the problem is stated in the most general case, leads us to find some non-conventional configurations, which show better damping properties when compared to the classical constrained layer treatments, namely classical hybrid elastomer/composite laminates.

Another point deserves attention: the passive solution consisting in patches bonded over composite plates is a good alternative with respect to introducing viscoelastic layers in sandwich plates, especially in terms of manufacturing because the patches can be deposited a posteriori on the plate surface. Nevertheless, we can see by the example treated in this paper, that such technology is relevant only if the structure is optimised $a$ priori, i.e. if the simultaneous design of both elastic and viscoelastic properties of the structure is taken into account.

The proposed approach appears to be very flexible and applicable to various problems of structural engineering. Moreover, the procedure has a high level of versatility: more constraints could be easily added to the optimisation problem, e.g. constraints on the strength, yielding or de-lamination of the laminate, without reducing the power and the robustness of the proposed approach.

\section{Acknowledgements}

The first author is grateful to the National Research Fund (FNR) in Luxembourg, for supporting this research work through Aides à la Formation Recherche Grant (PHD-09-139). 


\section{References}

Araújo, A., Martins, P., Mota Soares, C., Mota Soares, C. and Herskovits, J. (2009). Damping optimization of viscoelastic laminated sandwich composite structures, Structural and Multidisciplinary Optimization 39: $569-579$.

Araújo, A., Martins, P., Mota Soares, C., Mota Soares, C. and Herskovits, J. (2012). Damping optimisation of hybrid active-passive sandwich composite structures, Advances in Engineering Software 46: 69-74.

Araújo, A., Mota Soares, C., Mota Soares, C. and Herskovits, J. (2010). Optimal design and parameter estimation of frequency dependent viscoelastic laminated sandwich composite plates, Composite Structures 92: 2321-2327.

Bendsøe, M., Daz, A., Lipton, R. and Taylor, J. (1995). Optimal design of material properties and material distribution for multiple loading conditions, International Journal for Numerical Methods in Engineering 38(7): 1149-1170.

Benjeddou, A. (2001). Advances in hybrid active-passive vibration and noise control via piezoelectric and viscoelastic constrained layer treatments, Journal of Vibration and Control 7: 565-602.

Berthelot, J. M., Assarar, M., Sefrani, Y. and Mahi, A. E. (2008). Damping analysis of composite material and structures, Composite Structures 85: 189-204.

Chalak, H., Chakrabarti, A., Iqbal, M. and Sheikh, A. (2011). Vibration of laminated sandwich beams having soft core, Journal of Vibration and Control 18(10): 1422-1435.

Chandra, R., Singh, S. P. and Gupta, K. (1999). Damping studies in fiber-reinforced composites - a review, Composite Structures 46: 41-45.

Ghoneim, H. and Karkoub, M. (2001). Active, shunted, and passive constrained layer damping for the vibration suppression of a flexible four-bar mechanism, Journal of Vibration and Control 7: 551-563.

Hörnlein, H., Kocvara, M. and Werner, R. (2001). Material optimization: bridging the gap between conceptual and preliminary design, Aerospace Science and Technology 5(8): 541-554.

Hvejsel, C. F., Lund, E. and Stolpe, M. (2011). Optimization strategies for discrete multi-material stiffness optimization, Structural and Multidisciplinary Optimization 44: 149-163.

Julien, C. (2010). Conception optimale de l'anisotropie dans les structures stratifiées à rigidité variable par la mèthode polaire-génétiques (Optimal design of the anisotropy of variable-stiffness laminated structures through the polar-genetic method) (in French), PhD thesis, Institut d'Alembert UMR7190 CNRS -Université Pierre et Marie Curie Paris 6 , France.

Kerwin, E. M. (1959). Damping of flexural waves by a constrained viscoelastic layer, Journal of the Acoustic Society of America 37(7): 952-962.

Kristensen, R. F., Nielsen, K. L. and Mikkelsen, L. P. (2008). Numerical studies of shear damped composite beams using a constrained damping layer, Composite structures 83: 304-311.

Le Maoût, N., Verron, E. and Bégué, J. (2011). On the use of discontinuous elastomer patches to optimize the damping properties of composite sandwich plates, Composite Structures 93: 3057-3062.

Lu, Y. P., Killian, J. W. and Everstine, G. C. (1979). Vibrations of three layered damped sandwich plate composites, Journal of Sound and Vibration 62(1): 63-71.

Montemurro, M., Koutsawa, Y., Belouettar, S., Vincenti, A. and Vannucci, P. (2012). Design of damping properties of hybrid laminates through a global optimisation strategy, Composite Structures 94: 33093320.

Montemurro, M., Vincenti, A. and Vannucci, P. (2012a). A two-level procedure for the global optimum design of composite modular structures - application to the design of an aircraft wing. Part 1: theoretical formulation, Journal of Optimization Theory and Applications 155(1): 1-23.

Montemurro, M., Vincenti, A. and Vannucci, P. (2012b). A two-level procedure for the global optimum design of composite modular structures - application to the design of an aircraft wing. Part 2: numerical aspects and examples, Journal of Optimization Theory and Applications 155(1): 24-53. 
Montemurro, M., Vincenti, A. and Vannucci, P. (2013). The automatic dynamic penalisation method (ADP) for handling constraints with genetic algorithms, Computer Methods in Applied Mechanics and Engineering 256: 70-87.

Nakra, B. C. (1998). Vibration control in machines and structures using viscoelastic damping, Journal of Sound and Vibration 211(3): 449-465.

Rao, D. K. (1978). Frequency and loss factor of sandwich beams under various boundary conditions, Journal of Mechanical Engineering Science 20(5): 271-282.

Sher, B. and Moreira, R. (2013). Dimensionless analysis of constrained damping treatments, Composite Structures 99: 241-254.

Trinidade, M. and Benjeddou, A. (2002). Hybrid active-passive damping treatments using viscoelastic and piezoelectric materials: Review and assessment, Journal of Vibration and Control 8: 699-745.

Vannucci, P. (2005). Plane anisotropy by the polar method, Meccanica 40: 437-454.

Vannucci, P. (2006). Designing the elastic properties of laminates as an optimisation problem: a unified approach based on polar tensor invariants, Structural and Multidisciplinary Optimisation 31(5): 378387.

Vannucci, P. (2012). A note on the elastic and geometric bounds for composite laminates, Journal of Elasticity DOI: http://dx.doi.org/10.1007/s10659-012-9406-1 (In press).

Vannucci, P. and Verchery, G. (2001). Stiffness design of laminates using the polar method, International Journal of Solids and Structures 38: 9281-9294.

Vasques, C. M. A., Moreira, R. A. S. and Dias Rodrigues, J. (2010a). Viscoelastic damping technologies - part I: modeling and finite element implementation, Journal of Advanced Research in Mechanical Engineering 1: $76-95$.

Vasques, C. M. A., Moreira, R. A. S. and Dias Rodrigues, J. (2010b). Viscoelastic damping technologies part II: experimental identification procedure and validation, Journal of Advanced Research in Mechanical Engineering 1(2): 96-110.

Verchery, G. (1979). Les invariants des tenseurs d'ordre 4 du type de l'élasticité (The invariants of fourth-order elasticity-like tensors), Proceedings of the Euromech Colloquium 115, VIllard-de-Lans, (France).

Vincenti, A., Ahmadian, M. R. and Vannucci, P. (2010). BIANCA: a genetic algorithm to solve hard combinatorial optimisation problems in engineering, Journal of Global Optimisation 48: 399-421.

Vincenti, A., Vannucci, P. and Ahmadian, M. R. (2012 (In press)). Optimization of laminated composites by using genetic algorithm and the polar description of plane anisotropy, Mechanics of Advanced Materials and Structures p. http://dx.doi.org/10.1080/15376494.2011.563415.

Zhang, S. H. and Chen, H. L. (2006). A study on the damping characteristics of laminated composites with integral viscoelastic layers, Composite Structures 74: 63-69.

Zheng, H., Cai, C. and Tan, X. M. (2004). Optimization of partial constrained layer damping treatment for vibrational energy minimization of vibrating beams, Computers \&3 Structures 82: 2493-2507. 